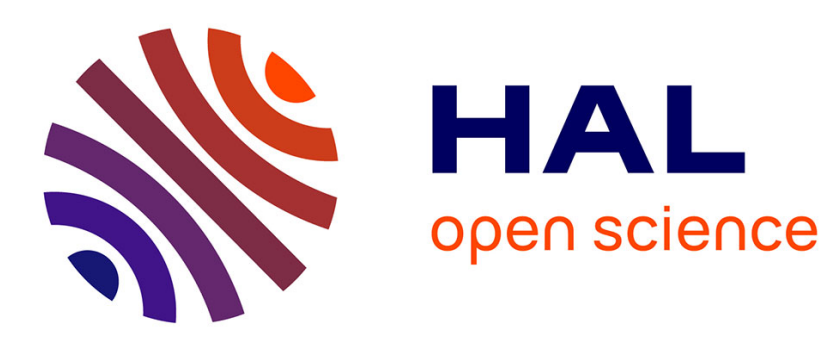

\title{
Topology optimization for maximizing the fracture resistance of quasi-brittle composites
}

\author{
Liang Xia, Daicong Da, Julien Yvonnet
}

\section{To cite this version:}

Liang Xia, Daicong Da, Julien Yvonnet. Topology optimization for maximizing the fracture resistance of quasi-brittle composites. Computer Methods in Applied Mechanics and Engineering, 2018, 332, pp.234-254. 10.1016/j.cma.2017.12.021 . hal-01674145

\section{HAL Id: hal-01674145 \\ https://hal.science/hal-01674145}

Submitted on 1 Jan 2018

HAL is a multi-disciplinary open access archive for the deposit and dissemination of scientific research documents, whether they are published or not. The documents may come from teaching and research institutions in France or abroad, or from public or private research centers.
L'archive ouverte pluridisciplinaire HAL, est destinée au dépôt et à la diffusion de documents scientifiques de niveau recherche, publiés ou non, émanant des établissements d'enseignement et de recherche français ou étrangers, des laboratoires publics ou privés. 


\title{
Topology optimization for maximizing the fracture resistance of quasi-brittle composites
}

\author{
Liang $\mathrm{Xia}^{\mathrm{a}, \mathrm{b}, *}$, Daicong $\mathrm{Da}^{\mathrm{b}, \mathrm{c}}$, Julien Yvonnet ${ }^{\mathrm{b}}$ \\ ${ }^{a}$ State Key Laboratory of Digital Manufacturing Equipment and Technology, \\ Huazhong University of Science and Technology, Wuhan 430074, China \\ ${ }^{b}$ Université Paris-Est, Laboratoire Modélisation et Simulation Multi Echelle MSME, \\ UMR CNRS 8208, 5 bd Descartes, 77454 Marne-la-Vallée, France \\ ' State Key Laboratory of Advanced Design and Manufacturing for Vehicle Body, \\ Hunan University, Changsha 410082, China
}

\begin{abstract}
In this paper, we propose a numerical framework for optimizing the fracture resistance of quasibrittle composites through a modification of the topology of the inclusion phase. The phase field method to fracturing is adopted within a regularized description of discontinuities, allowing to take into account cracking in regular meshes, which is highly advantageous for topology optimization purpose. Extended bi-directional evolutionary structural optimization (BESO) method is employed and formulated to find the optimal distribution of inclusion phase, given a target volume fraction of inclusion and seeking a maximal fracture resistance. A computationally efficient adjoint sensitivity formulation is derived to account for the whole fracturing process, involving crack initiation, propagation and complete failure of the specimen. The effectiveness of developed framework is illustrated through a series of $2 \mathrm{D}$ and $3 \mathrm{D}$ benchmark tests.
\end{abstract}

Keywords: Fracture resistance, Topology optimization, BESO, Phase field method, Crack propagation

\section{Introduction}

Composite materials are usually made of two or more constituent materials with variant mechanical properties and have advantageous overall characteristics when compared to traditional materials. The overall behavior of composite materials, such as fibrous composite, concrete, metallic porous material and metal alloy, depends strongly on the size, shape, spatial distribution and properties of the constituents. Seeking for an optimal design of spatial distribution of the constituents in heterogeneous media has recently attracted a growing attention (see e.g., [1-4]).

Among all properties of interest, accounting for material failure is of essential importance in the design of composite materials. As illustrated in Fig. 1, it is desired to improve the fracture

\footnotetext{
${ }^{*}$ Corresponding author

Email address: xialiang@hust.edu.cn (Liang Xia)
} 

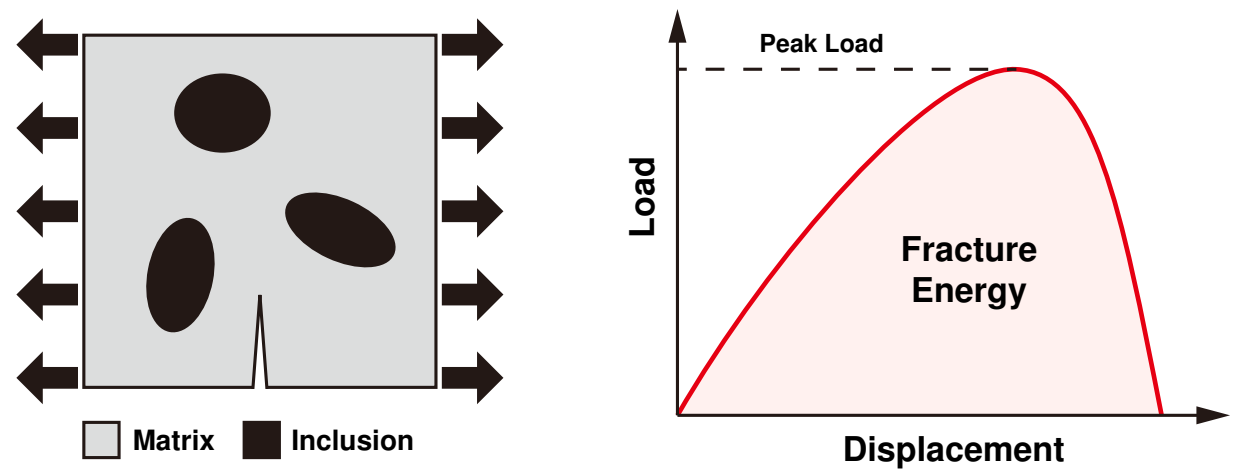

Figure 1: Illustration of the enforcement of a pre-cracked composite for fracture resistance.

resistance in terms of the required mechanical work for complete failure through an optimal placement of the inclusion phase. Due to the modeling complexity of fracturing, it is still an unexplored field upon the literature. Prechtel et al. [5] investigated on fiber-reinforced composites and adopted a gradient-free pattern search algorithm for the design of fiber shapes such that the fracture energy required to release for complete failure is maximized, in which a cohesive model is used for the cracking simulation. Gu et al. [6] designed the optimal layout distribution of stiff inclusions within a soft matrix using a gradient-free greedy search algorithm for various fracture resistant properties under uniaxial tension, whereas the simulation was realized using the finite element method without accounting for the crack propagation. More recently, San and Waisman [7] used a genetic algorithm to find optimal placement of reinforcing particles to maximize the fracture resistance of carbon black polymer, in which the phase field method was used for cracking simulation accounting for meanwhile large deformation kinematics.

One common feature of aforementioned works is that only a limited number of design variables was considered, which allows the use of gradient-free optimization algorithms. However, such gradient-free optimization algorithms are no longer applicable when it comes to a larger number of design variables as in the highly demanding computational requirements of fracturing simulation. To this end, we develop in this work a topology optimization framework for fracture resistance of quasi-brittle composite structures through an optimal design of the spatial distribution of inclusion phase. On the one hand, unlike previous works where inclusion shapes or layout typologies are a priori fixed, topology optimization adopted in the present work provides new design possibilities. On the other hand, topology optimization design variables are updated upon gradient information, which is highly efficient in terms of required design iterations as compared to gradient-free algorithms.

As an advanced design tool, topology optimization has undergone a remarkable development since the seminal paper by Bendsøe and Kikuchi [8] over the past decades in both academic research [9, 10] and industrial applications [11]. By topology optimization, one aims to find an optimal material layout within a prescribed design domain so as to maximize or minimize certain objectives and satisfying one or multiple design constraints. The key merit of topology optimization over conventional sizing or shape optimizations is that the structural topology or the material layout inside the design domain is not a priori assumed, resulting in more freedom about the de- 
sign and in most case to more efficient performances. Even though there do exist quite numerous researches on topology optimization with the enforcement of stress constraints (e.g., [12-20]) and damage constraints (e.g., [21-31]), topology optimization for fracture resistance accounting for complete fracturing process has not been explored in the literature to our best knowledge.

In this work, the extended bi-directional evolutionary structural optimization (BESO) method recently developed by the first author and his collaborators in [32,33] for the design of elastoplastic structures is adopted to carry out topology optimization. As compared to continuously defined density-based methods, the ESO-type methods [34-38] naturally avoid the definition of supplementing pseudo-relationships between fictitious materials and fracture toughness for the sake of their discrete nature, resulting in clear physical interpretation and algorithmic advantages. For a prescribed material volume usage of inclusion phase, the extended BESO method is adopted to seek for an optimal spatial distribution of inclusion phase so as to maximize the fracture resistance of composites. A computationally efficient adjoint sensitivity formulation is derived that accounts for the complete fracturing process.

With regard to fracturing simulation, the phase field approach proposed in Francfort and Marigo [39], Bourdin et al. [40], Hakim and Karma [41], Miehe et al. [42, 43], Borden et al. [44] (only to name a few) is adopted for its computational advantages in handling very complex crack topologies. It should be noted that the phase field approach to fracture is consistent with brittle fracture through variational methods based on energy minimization as shown in Francfort and Marigo [39]. In this framework, the sharp crack discontinuities are regularized by a diffuse phase field approximation within a continuum formulation, making it very flexible to handle crack nucleation, multiple crack fronts, cracks merging and branching in both $2 \mathrm{D}$ and 3D without ad hoc numerical treatment (see e.g. [42, 43, 45-48]). In addition, due to its gradient-based formulation, it is not sensitive to the mesh orientation and thus regular meshes can then be used, which is crucial in topological optimization procedures where regular meshes are most often employed.

This paper is organized as follows. Section 2 first reviews the phase field method for the modeling of crack propagation as developed by Miehe et al. [43, 45]. Section 3 presents the topology optimization method for design of quasi-brittle composites with fracture resistance. Section 4 validates the proposed design framework through a series of 2D and 3D benchmark tests. Finally, conclusion and future perspectives are drawn.

\section{Phase field modeling of crack propagation}

Let $\Omega \in \mathbb{R}^{D}$ be an open domain describing a cracked solid as depicted in Fig. 2 , with $D \in$ $[2,3]$ being the space dimension. The external boundary of $\Omega$ is denoted by $\partial \Omega \in \mathbb{R}^{D-1}$. Cracks which may propagate within the solid are collectively denoted by $\Gamma$. In this work, we adopt the framework proposed in $[42,43,45,49,50]$ for a regularized representation of discontinuities. In this regularized framework, the propagating cracks are approximately represented by an evolving scalar phase field $d(\mathbf{x}, t)$, where the diffusion is characterized by a length scale parameter $\ell$.

\subsection{Phase field approximation of cracks}

The scalar crack phase field $d(\mathbf{x}, t)$ can be determined through solving the following boundary value problem subject to Dirichlet boundary conditions $(d=1)$ on the crack (see [42] for more 


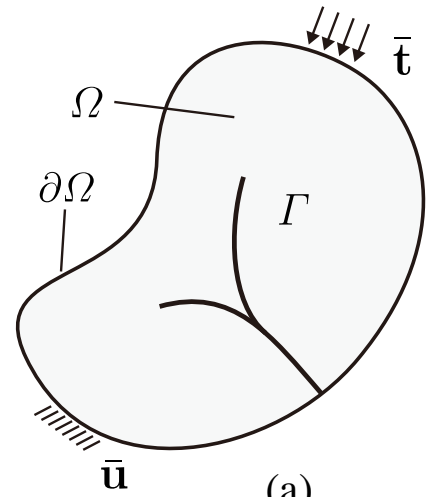

(a)

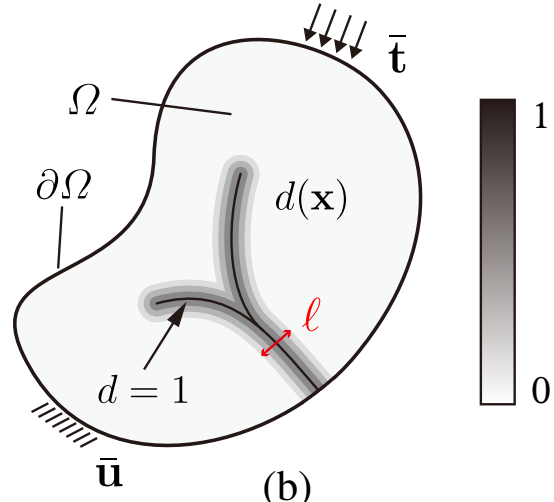

(b)

Figure 2: Illustration of phase field crack modeling: (a) a sharp crack surface $\Gamma$ embedded into the solid $\Omega$; (b) the regularized representation of the crack by the phase field $d(\mathbf{x})$.

details):

$$
\left\{\begin{array}{l}
d(\mathbf{x}, t)-\ell^{2} \nabla^{2} d(\mathbf{x}, t)=0, \text { in } \Omega \\
d(\mathbf{x}, t)=1, \text { on } \Gamma \\
\nabla d(\mathbf{x}, t) \cdot \mathbf{n}=0 \text { on } \partial \Omega,
\end{array}\right.
$$

where $\nabla^{2}($.$) is the Laplacian operator, \ell$ is a length scale parameter that governs the width of the regularization zone and gives for $\ell \rightarrow 0$ the exact sharp crack in $\Gamma$ in (1), and $\mathbf{n}$ is the unit outward normal vector to $\partial \Omega$. In the following, we denote the crack phase field $d(\mathbf{x}, t)$ by $d$ to alleviate the notations. It has been shown that the system of equations (1) corresponds to the Euler-Lagrange equation associated with the variational problem:

$$
d=\operatorname{Arg}\left\{\inf _{d \in S} \Gamma(d)\right\}, \quad \Gamma(d)=\int_{\Omega} \gamma(d) \mathrm{d} V,
$$

where $S=\{d \mid d(\mathbf{x})=1, \forall \mathbf{x} \in \Gamma\}$, and $\gamma$ is the crack surface density function per unit volume defined by:

$$
\gamma(d)=\frac{1}{2 \ell} d^{2}+\frac{\ell}{2} \nabla d \cdot \nabla d
$$

The functional $\Gamma(d)$ represents the total length of the crack in 2D and the total crack surface area in 3D. A detailed explanation of (3) can be found in [42].

\subsection{Thermodynamics of the phase field crack evolution}

The variational approach to fracture mechanics provided by Francfort and Marigo [39] introduces the following energy functional for a cracked body:

$$
J(\mathbf{u}, \Gamma)=\int_{\Omega} W_{u}(\boldsymbol{\varepsilon}(\mathbf{u})) \mathrm{d} V+\int_{\Gamma} g_{c} \mathrm{~d} A,
$$

in which $W_{u}$ is the energy density function where $\varepsilon=\frac{1}{2}\left(\nabla \mathbf{u}+\nabla^{T} \mathbf{u}\right)$ the strain and $\mathbf{u}$ the displacement field. The first term on the right hand side of (4) corresponds to the elastic energy stored in the 
cracked solid. The second term on the right hand side of (4) corresponds to the energy required to create the crack according to the Griffith criterion with $g_{c}$ the critical fracture energy density, also named as Griffith's critical energy release rate.

In the adopted regularized framework, the phase field $d(\mathbf{x})$ is introduced for the representation of cracks. Then above functional (4) is substituted by the following one:

$$
J(\mathbf{u}, d)=\int_{\Omega} W_{u}(\boldsymbol{\varepsilon}, d) \mathrm{d} V+\int_{\Omega} g_{c} \gamma(d) \mathrm{d} V,
$$

where $\gamma(d)$ is the surface density defined in (3). From (5), the energy potential or free energy $W$ can be identified as:

$$
W(\boldsymbol{\varepsilon}, d)=W_{u}(\boldsymbol{\varepsilon}, d)+g_{c} \gamma(d) .
$$

Following [42], the elastic energy $W_{u}$ is defined in the following form that assumes isotropic elastic behavior of the solid and accounts for damage induced by traction only, through:

$$
W_{u}(\boldsymbol{\varepsilon}, d)=\left((1-d)^{2}+\kappa\right) \psi^{+}(\boldsymbol{\varepsilon})+\psi^{-}(\boldsymbol{\varepsilon}),
$$

where $\kappa \ll 1$ is a small positive parameter introduced to prevent the singularity of the stiffness matrix due to fully broken parts, $\psi^{+}$and $\psi^{-}$are the the tensile and compressive strain energies,

$$
\psi^{ \pm}=\lambda\langle\operatorname{tr}[\varepsilon]\rangle_{ \pm}^{2} / 2+\mu \operatorname{tr}\left[\varepsilon^{ \pm}\right]^{2}
$$

with $\lambda$ and $\mu$ the Lamé coefficients of the solid. Only tensile damage degradation is taken into account in the elastic energy (7) through a decomposition of the elastic strain $\varepsilon$ into tensile and compressive parts [42]:

$$
\boldsymbol{\varepsilon}=\boldsymbol{\varepsilon}^{+}+\boldsymbol{\varepsilon}^{-} \quad \text { with } \quad \boldsymbol{\varepsilon}^{ \pm}=\sum_{i=1}^{3}\left\langle\varepsilon^{i}\right\rangle_{ \pm} \mathbf{n}^{i} \otimes \mathbf{n}^{i}
$$

In the above, $\langle x\rangle_{ \pm}=(x \pm|x|) / 2$, and $\varepsilon^{i}$ and $\mathbf{n}^{i}$ are the eigenvalues and eigenvectors of $\boldsymbol{\varepsilon}$. The evolution of the damage variable $d(\mathbf{x}, t)$ can be determined by the variational derivative of the free energy $W$. In a rate-independent setting with the consideration of the reduced Clausius-Duhem inequality, the evolution criterion is provided by the Kuhn-Tucker conditions [42, 46]:

$$
\dot{d} \geq 0 ; \quad-\delta_{d} W \leq 0 ; \quad \dot{d}\left[-\delta_{d} W\right]=0,
$$

yielding

$$
-\delta_{d} W=2(1-d) \psi^{+}(\varepsilon)-g_{c} \delta_{d} \gamma=0,
$$

with the functional derivative [42]

$$
\delta_{d} \gamma=d / \ell-\ell \Delta d
$$

Following [43], the maximum tensile strain energy is stored to account for loading and unloading histories and the damage evolution criterion (11) can then be expressed in the following form:

$$
\frac{g_{c}}{\ell}\left[d-\ell^{2} \nabla^{2} d\right]=2(1-d) \max _{t \in[0, T]}\left\{\psi^{+}(\mathbf{x}, t)\right\} .
$$


The criterion (13) is a monotonously increasing function of the strain $\boldsymbol{\varepsilon}(\mathbf{x}, t)$ that induces unnecessary stress degradation even at low strain values. To avoid this issue, an energetic damage evolution criterion with threshold has been introduced in [45, 51, 52], yielding

$$
\psi_{c}\left[d-\ell^{2} \nabla^{2} d\right]=(1-d) \max _{t \in[0, T]}\left\{\left\langle\psi^{+}(\mathbf{x}, t)-\psi_{c}\right\rangle_{+}\right\}
$$

in which $\psi_{\mathrm{c}}$ is a specific fracture energy density of the solid, which can be further related to a critical fracture stress $\sigma_{c}$ by:

$$
\psi_{c}=\frac{1}{2 E} \sigma_{c}^{2},
$$

where $E$ is the Young's modulus (see more details in [45]). The above crack evolution criterion (14) can be further stated as

$$
\psi_{c}\left[d-\ell^{2} \nabla^{2} d\right]=(1-d) \mathcal{H}(\mathbf{x}, t)
$$

with the introduction of a strain energy history function [52]

$$
\mathcal{H}(\mathbf{x}, t)=\max _{t \in[0, T]}\left\{\left\langle\psi^{+}(\mathbf{x}, t)-\psi_{c}\right\rangle_{+}\right\}
$$

\subsection{Weak forms of displacement and phase field problems} reads

In the absence of body forces, the linear momentum balance equation for the solid medium

$$
\nabla \cdot \sigma=\mathbf{0}
$$

where according to the definitions in (7) and (8), the stress tensor $\sigma$ equals

$$
\boldsymbol{\sigma}=\frac{\partial W_{u}}{\partial \boldsymbol{\varepsilon}}=\left((1-d)^{2}+\kappa\right)\left(\lambda\langle\operatorname{tr}[\boldsymbol{\varepsilon}]\rangle_{+} \mathbf{1}+2 \mu \boldsymbol{\varepsilon}^{+}\right)+\lambda\langle\operatorname{tr}[\boldsymbol{\varepsilon}]\rangle_{-} \mathbf{1}+2 \mu \boldsymbol{\varepsilon}^{-},
$$

in which $\mathbf{1}$ is the second-order identity tensor and $\kappa \ll 1$ is a small positive parameter introduced to prevent the singularity of the stiffness matrix due to fully broken parts. Multiplying the governing equation (18) by kinematically admissible test functions for the displacement $\delta \mathbf{u}$, integrating the resulting expression over the domain $\Omega$, and using the divergence theorem together with boundary conditions yields the associated weak form:

$$
\int_{\Omega} \boldsymbol{\sigma}: \boldsymbol{\varepsilon}(\delta \mathbf{u}) \mathrm{d} V=\int_{\partial \Omega_{t}} \overline{\mathbf{t}} \cdot \delta \mathbf{u} \mathrm{d} A,
$$

in which $\overline{\mathbf{t}}$ is the applied traction on the Neumann boundary $\partial \Omega_{t}$ (see Fig. 2). The weak form (20) is completed with Dirichlet boundary conditions defined on $\partial \Omega_{u}$.

The associated weak form for the crack phase field evolution (16) can be obtained in a similar fashion:

$$
\int_{\Omega}\left\{\left(\mathcal{H}+\psi_{c}\right) d \delta d+\psi_{c} \ell^{2} \nabla d \cdot \nabla(\delta d)\right\} \mathrm{d} V=\int_{\Omega} \mathcal{H} \delta d \mathrm{~d} V
$$

in which $\delta d \in H_{0}^{1}(\Omega), d \in H^{1}(\Omega)$ and satisfying the Dirichlet boundary conditions on $\Gamma$. 


\subsection{Finite element discretization}

In this work, we adopt the same finite element discretization for the approximation of the displacement field $\mathbf{u}$ and the crack phase field $d$. We can express the two finite element approximate fields $\left(\mathbf{u}^{h}, d^{h}\right)$ as:

$$
\mathbf{u}^{h}(\mathbf{x})=\mathbf{N}_{u}(\mathbf{x}) \mathbf{d}_{u}, d^{h}(\mathbf{x})=\mathbf{N}_{d}(\mathbf{x}) \mathbf{d}_{d}
$$

and their gradients as

$$
\nabla \mathbf{u}^{h}(\mathbf{x})=\mathbf{B}_{u}(\mathbf{x}) \mathbf{d}_{u}, \nabla d^{h}(\mathbf{x})=\mathbf{B}_{d}(\mathbf{x}) \mathbf{d}_{d},
$$

where $\mathbf{N}_{u}$ and $\mathbf{B}_{u}$ denote matrices of shape functions and shape functions derivatives associated to displacements, and $\mathbf{N}_{d}$ and $\mathbf{B}_{d}$ denote matrices of shape functions and shapre functions derivatives associated to phase field variable. Here, $\left\{\mathbf{d}_{u}, \mathbf{d}_{d}\right\}$ denote the vectors of the nodal values of the finite element mesh for displacement and crack phase fields, respectively.

Introducing the above discretization into the weak form (20), we obtain the following discrete system of equations:

$$
\mathbf{K}_{u} \mathbf{d}_{u}=\mathbf{f}_{u},
$$

with the external force vector $\mathbf{f}_{u}$

$$
\mathbf{f}_{u}=\int_{\partial \Omega_{t}} \mathbf{N}_{u} \overline{\mathbf{t}} \mathrm{d} A
$$

and the stiffness matrix $\mathbf{K}_{u}$

$$
\mathbf{K}_{u}=\int_{\Omega} \mathbf{B}_{u}^{T} \mathbf{D} \mathbf{B}_{u} \mathrm{~d} V
$$

where $\mathbf{D}$ is the constitutive matrix corresponding to the definition in (19), given by:

$$
\mathbf{D}=\frac{\partial[\boldsymbol{\sigma}]}{\partial[\boldsymbol{\varepsilon}]}=(1-d)^{2}\left(\lambda \mathcal{R}^{+}[\mathbf{1}][\mathbf{1}]^{T}+2 \mu \mathbf{P}^{+}\right)+\left(\lambda \mathcal{R}^{-}[\mathbf{1}][\mathbf{1}]^{T}+2 \mu \mathbf{P}^{-}\right)
$$

where $[\sigma]$ and $[\varepsilon]$ are the vector forms corresponding to the second order tensors of stress $\sigma$ and strain $\boldsymbol{\varepsilon}$. $\mathcal{R}^{ \pm}$and $\mathbf{P}^{ \pm}$are two operators for the decomposition of strain into the tensile and compressive parts (see e.g., [46]). The matrices $\mathbf{P}^{ \pm}$are such that:

$$
\left[\varepsilon^{+}\right]=\mathbf{P}^{+}[\varepsilon] \text { and }\left[\varepsilon^{-}\right]=\mathbf{P}^{-}[\varepsilon] .
$$

The discretization of the phase field problem (21) leads to the following discrete system of equations:

$$
\mathbf{K}_{d} \mathbf{d}_{d}=\mathbf{f}_{d}
$$

where

$$
\mathbf{K}_{d}=\int_{\Omega}\left\{\left(\mathcal{H}+\psi_{c}\right) \mathbf{N}_{d}^{T} \mathbf{N}_{d}+\psi_{c} \ell^{2} \mathbf{B}_{d}^{T} \mathbf{B}_{d}\right\} \mathrm{d} V
$$

and

$$
\mathbf{f}_{d}=\int_{\Omega} \mathbf{N}_{d}^{T} \mathcal{H} \mathrm{d} V
$$

in which $\mathcal{H}$ is strain energy history function defined in (17). 
In the present work, a staggered solution scheme is employed following [43], where at each time increment the phase field problem is solved for fixed displacement field known from the previous time step $t^{n}$. The mechanical problem is then solved given the phase field at the new time step $t^{n+1}$. The overall algorithm is described as follows:

1. Set the initial fields $d\left(t_{0}\right), \mathbf{u}\left(t_{0}\right)$, and $\mathcal{H}\left(t_{0}\right)$ at time $t_{0}$.

2. Loop over all time increments: at each time $t_{n+1}$ :

(a) Given $d\left(t_{n}\right), \mathbf{u}\left(t_{n}\right)$, and $\mathcal{H}\left(t_{n}\right)$ :

(b) Compute the history function $\mathcal{H}\left(t_{n+1}\right)$ according to (17).

(c) Compute the crack phase field $d\left(t_{n+1}\right)$ by solving (29).

(d) Compute $\mathbf{u}\left(t_{n+1}\right)$ with the current crack $d\left(t_{n+1}\right)$ by solving (24).

(e) $(.)_{n} \leftarrow(.)_{n+1}$ and go to (a).

\section{End.}

\section{Topology optimization model for fracture resistance}

The extended bi-directional evolutionary structural optimization (BESO) method developed in $[32,33]$ for the design of elastoplastic structures is adopted in this work to carry out topology optimization. Composites made of two material phases, matrix phase and inclusion phase, are considered. The spatial layout of inclusion phase is optimized by the extended BESO method to yield composite with a higher fracture resistance.

\subsection{Model definitions}

The design domain $\Omega$ is discretized into $N_{\mathrm{e}}$ finite elements and each element $e$ is assigned with a topology design variable $\rho_{e}$. The $N_{\mathrm{e}}$-dimensional vector containing the design variables is denoted as $\boldsymbol{\rho}=\left(\rho_{1}, \ldots, \rho_{N_{\mathrm{e}}}\right)^{T}$. Following [53], the design variables and the multiple material interpolation model are defined as

$$
\rho_{e}=0 \text { or } 1, \quad e=1,2, \ldots, N_{\mathrm{e}}
$$

and

$$
\left\{\begin{array}{l}
E_{e}=\rho_{e} E_{\mathrm{inc}}+\left(1-\rho_{e}\right) E_{\mathrm{mat}} \\
\sigma_{c, e}=\rho_{e} \sigma_{c, \mathrm{inc}}+\left(1-\rho_{e}\right) \sigma_{c, \text { mat }},
\end{array}\right.
$$

where $E_{e}$ and $\sigma_{c, e}$ are the Young's modulus and the critical fracture stress of the $e$-th element. $\left\{E_{\text {inc }}, \sigma_{c \text {,inc }}\right\}$ and $\left\{E_{\text {mat }}, \sigma_{c \text {,mat }}\right\}$ are the Young's moduli and the critical fracture stresses of the inclusion and the matrix phases, respectively. Attention needs to be recalled that " $E_{\mathrm{inc}}>E_{\mathrm{mat}}$ " is assumed when carrying out topology optimization with multiple materials using the BESO method [53]. The Poisson's ratios of the two material phases are assumed identical. The design variables can thus be interpreted as an indicator such that the value of one corresponds to the inclusion phase, whereas zero corresponds to the matrix phase.

For stability considerations, it is conventionally to adopt displacement-controlled loading for nonlinear designs (e.g., [32, 33, 54-56]). For a prescribed displacement load, the fracture resistance maximization is equivalent to the maximization of the mechanical work expended in the 
course of the fracturing process as illustrated in Fig. 1. In practice, the total mechanical work $J$, is approximated by numerical integration using the trapezoidal rule, i.e.

$$
J \approx \frac{1}{2} \sum_{i=1}^{n_{\text {load }}}\left(\mathbf{f}_{u}^{(i)}+\mathbf{f}_{u}^{(i-1)}\right)^{T} \Delta \mathbf{d}_{u}^{(i)}
$$

Here $n_{\text {load }}$ is the total number of displacement increments, $\Delta \mathbf{d}_{u}^{(i)}$ is the $i$-th increment of the nodal displacement vector and $\mathbf{f}_{u}^{(i)}$ is the external nodal force vector (comprising surface tractions and reaction forces) at the $i$-th load increment.

During the optimization process the volume fraction of the inclusion phase is prescribed. Then the optimization problem discretized with $N_{\mathrm{e}}$ elements can be formulated as [e.g., 32, 33, 56]

$$
\begin{aligned}
\max _{\boldsymbol{\rho}}: & J\left(\boldsymbol{\rho}, \mathbf{d}_{u}, \mathbf{d}_{d}\right) \\
\text { subject to : } & \mathbf{K}_{u}^{(i)} \mathbf{d}_{u}^{(i)}=\mathbf{f}_{u}^{(i)}, i=1, \ldots, n_{\text {load }} \\
: & V(\boldsymbol{\rho})=\sum \rho_{e} v_{e}=V_{\text {req }} \\
: & \rho_{e}=0 \text { or } 1, e=1, \ldots, N_{\mathrm{e}} .
\end{aligned}
$$

Here $v_{e}$ is the volume of the $e$-th element, $V(\rho)$ and $V_{\text {req }}$ are the total and required material volumes, respectively. The stiffness matrix $\mathbf{K}_{u}^{(i)}$ at the $i$-th load increment is constructed following (26) and (27).

It may be recalled that by this model the discrete topology design variable $\rho_{e} \in\{0 ; 1\}$ indicates merely the associated material phase (matrix/inclusion) of the $e$-th element. This assumption omits naturally the definition of supplementing pseudo-relationships between intermediate densities and their constitutive behaviors as is the case in density-based models (e.g., [55, 57, 58]) resulting in algorithmic advantages (see also [32, 33]).

\subsection{Sensitivity analysis}

In order to perform the topology optimization, the sensitivity of the objective function $J$ with respect to topology design variables $\rho$ needs to be provided. Following the "hard-kill" BESO procedure $[36,53]$, the topology evolution is driven merely by the sensitivities of the solid phase ( $\rho=1$, the inclusion phase in the current context), whilst the sensitivities of the void phase with $(\rho=0$, the matrix phase) are set to zero.

The derivation of the sensitivity requires using the adjoint method (see, e.g., $[59,60]$ ). Lagrange multipliers $\boldsymbol{\mu}^{(i)}, \lambda^{(i)}$ of the same dimension as the vector of unknowns $\mathbf{d}_{u}$ are introduced in order to enforce zero residual $\mathbf{r}$ at times $t_{i-1}$ and $t_{i}$ for each term of the quadrature rule (34). Then the objective function $J$ can be rewritten in the following form without modifying the original objective value as

$$
J^{*}=\frac{1}{2} \sum_{i=1}^{n_{\text {load }}}\left\{\left(\mathbf{f}_{u}^{(i)}+\mathbf{f}_{u}^{(i-1)}\right)^{T} \Delta \mathbf{d}_{u}^{(i)}+\left(\lambda^{(i)}\right)^{T} \mathbf{r}^{(i)}+\left(\boldsymbol{\mu}^{(i)}\right)^{T} \mathbf{r}^{(i-1)}\right\} .
$$

Due to the asserted static equilibrium the residuals $\mathbf{r}^{(i)}$ and $\mathbf{r}^{(i-1)}$ have to vanish. The objective value is, thus, invariant with respect to the values of the Lagrange multipliers $\lambda^{(i)}$ and $\boldsymbol{\mu}^{(i)}(i=$ $\left.1, \ldots, n_{\text {load }}\right)$, i.e.

$$
J^{*}\left(\rho ;\left\{\lambda^{(i)}, \mu^{(i)}\right\}_{i=1, \ldots, n_{\text {load }}}\right)=J(\rho) .
$$


This equivalence holds also for the sensitivity with respect to changes of the topology design variable $\rho_{e}$ on element $e$

$$
\frac{\partial J^{*}}{\partial \rho_{e}}=\frac{\partial J}{\partial \rho_{e}} .
$$

In the following the derivative $\partial J^{*} / \partial \rho_{e}$ is computed with properly determined values of $\lambda^{(i)}$ and $\boldsymbol{\mu}^{(i)}$ leading to certain simplifications of the derivation. To formally describe these derivations, we introduce a partitioning of all degrees of freedom (DOF) into essential (index E; associated with Dirichlet boundary conditions) and free (index F; remaining DOF) entries. For a vector $\mathbf{w}$ and a matrix $\mathbf{M}$ we have

$$
\mathbf{w} \sim\left[\begin{array}{c}
\mathbf{w}_{\mathrm{E}} \\
\mathbf{w}_{\mathrm{F}}
\end{array}\right] \text { and } \mathbf{M} \sim\left[\begin{array}{ll}
\mathbf{M}_{\mathrm{EE}} & \mathbf{M}_{\mathrm{EF}} \\
\mathbf{M}_{\mathrm{FE}} & \mathbf{M}_{\mathrm{FF}}
\end{array}\right] .
$$

In the present context, the displacements $\mathbf{d}_{u, \mathrm{E}}$ on the Dirichlet boundary are prescribed and, hence, they are independent of the current value of $\rho$. This implies that

$$
\frac{\partial \Delta \mathbf{d}_{u}}{\partial \rho_{e}}=\frac{\partial}{\partial \rho_{e}}\left[\begin{array}{l}
\Delta \mathbf{d}_{u, \mathrm{E}} \\
\Delta \mathbf{d}_{u, \mathrm{~F}}
\end{array}\right]=\left[\begin{array}{c}
\mathbf{0} \\
\partial\left(\Delta \mathbf{d}_{u, \mathrm{~F}}\right) / \partial \rho_{e}
\end{array}\right]
$$

holds for arbitrary load time increments, i.e. for $\mathbf{d}_{u}=\mathbf{d}_{u}^{(i)}$ or $\mathbf{d}_{u}=\mathbf{d}_{u}^{(i-1)}$. The components $\mathbf{f}_{u, \mathrm{~F}}$ of the force vector $\mathbf{f}_{u}$ vanish at all load time increments and the only (possibly) non-zero components are the reaction forces $\mathbf{f}_{u, \mathrm{E}}$

$$
\mathbf{f}_{u}^{(i)}=\left[\begin{array}{c}
\mathbf{f}_{u, \mathrm{E}}^{(i)} \\
\mathbf{0}
\end{array}\right]
$$

Equations (40) and (41) imply

$$
\left(\mathbf{f}_{u}^{(j)}\right)^{T} \frac{\partial \Delta \mathbf{d}_{u}^{(i)}}{\partial \rho_{e}}=0
$$

Hence for arbitrary load increment indices $i, j=1, \ldots, n_{\text {load }}$, we have

$$
\frac{\partial}{\partial \rho_{e}}\left(\left(\mathbf{f}_{u}^{(j)}\right)^{T} \Delta \mathbf{d}_{u}^{(i)}\right)=\left(\frac{\partial \mathbf{f}_{u}^{(j)}}{\partial \rho_{e}}\right)^{T} \Delta \mathbf{d}_{u}^{(i)}
$$

With the property of (43) at hand, the derivative of the modified design objective in (38) is given by:

$$
\frac{\partial J^{*}}{\partial \rho_{e}}=\frac{1}{2} \sum_{i=1}^{n_{\text {load }}}\left\{\frac{\partial\left(\mathbf{f}_{u}^{(i)}+\mathbf{f}_{u}^{(i-1)}\right)^{T}}{\partial \rho_{e}} \Delta \mathbf{d}_{u}^{(i)}+\left(\lambda^{(i)}\right)^{T} \frac{\partial \mathbf{r}^{(i)}}{\partial \rho_{e}}+\left(\boldsymbol{\mu}^{(i)}\right)^{T} \frac{\partial \mathbf{r}^{(i-1)}}{\partial \rho_{e}}\right\} .
$$

Recall the equilibrium equation at each load time increment is given by (35), the derivatives of $\mathbf{r}^{(j)}$ at the equilibrium of the $j$-th load increment with respect to $\rho_{e}$ can be expanded as

$$
\frac{\partial \mathbf{r}^{(j)}}{\partial \rho_{e}}=\frac{\partial \mathbf{f}_{u}^{(j)}}{\partial \rho_{e}}-\frac{\partial \mathbf{K}_{u}^{(j)}}{\partial \rho_{e}} \mathbf{d}_{u}^{(j)}-\mathbf{K}_{u}^{(j)} \frac{\partial \mathbf{d}_{u}^{(j)}}{\partial \rho_{e}} .
$$


With the expression (45), (44) can be reformulated as

$$
\begin{aligned}
\frac{\partial J^{*}}{\partial \rho_{e}} & =\frac{1}{2} \sum_{i=1}^{n_{\text {load }}}\left\{\left(\frac{\partial \mathbf{f}_{u}^{(i)}}{\partial \rho_{e}}\right)^{T}\left(\Delta \mathbf{d}_{u}^{(i)}+\lambda^{(i)}\right)+\left(\frac{\partial \mathbf{f}_{u}^{(i-1)}}{\partial \rho_{e}}\right)^{T}\left(\Delta \mathbf{d}_{u}^{(i)}+\boldsymbol{\mu}^{(i)}\right)\right. \\
& \left.-\left(\lambda^{(i)}\right)^{T}\left(\frac{\partial \mathbf{K}_{u}^{(i)}}{\partial \rho_{e}} \mathbf{d}_{u}^{(i)}+\mathbf{K}_{u}^{(i)} \frac{\partial \mathbf{d}_{u}^{(i)}}{\partial \rho_{e}}\right)-\left(\boldsymbol{\mu}^{(i)}\right)^{T}\left(\frac{\partial \mathbf{K}_{u}^{(i-1)}}{\partial \rho_{e}} \mathbf{d}_{u}^{(i-1)}+\mathbf{K}_{u}^{(i-1)} \frac{\partial \mathbf{d}_{u}^{(i-1)}}{\partial \rho_{e}}\right)\right\} .
\end{aligned}
$$

As mentioned previously, the aim is to find proper values of the Lagrange multipliers $\lambda^{(i)}$ and $\boldsymbol{\mu}^{(i)}$ such that the sensitivities can be explicitly and efficiently computed. From the consideration of (41), the first two terms can be omitted by setting

$$
\lambda_{\mathrm{E}}^{(i)}=-\Delta \mathbf{d}_{u, \mathrm{E}}^{(i)} \quad \text { and } \quad \boldsymbol{\mu}_{\mathrm{E}}^{(i)}=-\Delta \mathbf{d}_{u, \mathrm{E}}^{(i)} .
$$

Accounting further for the structure of the sensitivities of $\mathbf{d}_{u}$ in (40) and for the symmetry of the stiffness matrices we have

$$
\begin{aligned}
\frac{\partial J^{*}}{\partial \rho_{e}}=-\frac{1}{2} \sum_{i=1}^{n_{\text {load }}}\{ & \left(\lambda^{(i)}\right)^{T} \frac{\partial \mathbf{K}_{u}^{(i)}}{\partial \rho_{e}} \mathbf{d}_{u}^{(i)}+\left(\mathbf{K}_{u, \mathrm{FE}}^{(i)} \lambda_{\mathrm{E}}^{(i)}+\mathbf{K}_{u, \mathrm{FF}}^{(i)} \lambda_{\mathrm{F}}^{(i)}\right)^{T} \frac{\partial \Delta \mathbf{d}_{u, \mathrm{~F}}^{(i)}}{\partial \rho_{e}} \\
& \left.+\left(\boldsymbol{\mu}^{(i)}\right)^{T} \frac{\partial \mathbf{K}_{u}^{(i-1)}}{\partial \rho_{e}} \mathbf{d}_{u}^{(i-1)}+\left(\mathbf{K}_{u, \mathrm{FE}}^{(i-1)} \boldsymbol{\mu}_{E}^{(i)}+\mathbf{K}_{u, \mathrm{FF}}^{(i-1)} \boldsymbol{\mu}_{F}^{(i)}\right)^{T} \frac{\partial \Delta \mathbf{d}_{u, \mathrm{~F}}^{(i-1)}}{\partial \rho_{e}}\right\} .
\end{aligned}
$$

In order to avoid the evaluation of the unknown derivatives of $\mathbf{d}_{u, \mathrm{~F}}^{(i)}$ and $\mathbf{d}_{u, \mathrm{~F}}^{(i-1)}$, the values of $\lambda_{\mathrm{F}}^{(i)}$ and $\mu_{\mathrm{F}}^{(i)}$ are sought as following by solving the adjoint systems with the prescribed values $\lambda_{\mathrm{E}}^{(i)}=-\Delta \mathbf{d}_{u, \mathrm{E}}^{(i)}$ and $\boldsymbol{\mu}_{\mathrm{E}}^{(i)}=-\Delta \mathbf{d}_{u, \mathrm{E}}^{(i)}$ at the essential nodes:

$$
\lambda_{\mathrm{F}}^{(i)}=\left(\mathbf{K}_{u, \mathrm{FF}}^{(i)}\right)^{-1} \mathbf{K}_{u, \mathrm{FE}}^{(i)} \Delta \mathbf{d}_{u, \mathrm{E}}^{(i)}
$$

and

$$
\boldsymbol{\mu}_{\mathrm{F}}^{(i)}=\left(\mathbf{K}_{u, \mathrm{FF}}^{(i-1)}\right)^{-1} \mathbf{K}_{u, \mathrm{FE}}^{(i-1)} \Delta \mathbf{d}_{u, \mathrm{E}}^{(i)} .
$$

The two relations (49) and (50) together with (47) completely determine the values of the Lagrange multipliers $\lambda^{(i)}$ and $\boldsymbol{\mu}^{(i)}$. It is obvious that the first adjoint system of (49) is in fact self-adjoint such that no additional calculation is needed and $\lambda^{(i)}=-\Delta \mathbf{d}_{u}^{(i)}$. Note in addition that because the proportional loading is increased at a constant rate, i.e.

$$
\Delta \mathbf{d}_{u, \mathrm{E}}^{(i)}=\frac{\Delta t^{(i)}}{\Delta t^{(i-1)}} \Delta \mathbf{d}_{u, \mathrm{E}}^{(i-1)}
$$

the solution of the second linear system (50) can also be omitted by means of the recursion formula

$$
\mu_{\mathrm{F}}^{(i)}=\frac{\Delta t^{(i)}}{\Delta t^{(i-1)}} \lambda_{\mathrm{F}}^{(i-1)}
$$


Substituting the two Lagrange multipliers into (48), the objective derivative $\partial J^{*} / \partial \rho_{e}$ can be eventually computed via

$$
\frac{\partial J^{*}}{\partial \rho_{e}}=\frac{1}{2} \sum_{i=1}^{n_{\text {load }}}\left\{\left(\lambda^{(i)}\right)^{T} \frac{\partial \mathbf{K}_{u}^{(i)}}{\partial \rho_{e}} \mathbf{d}_{u}^{(i)}+\left(\boldsymbol{\mu}^{(i)}\right)^{T} \frac{\partial \mathbf{K}_{u}^{(i-1)}}{\partial \rho_{e}} \mathbf{d}_{u}^{(i-1)}\right\}
$$

in which

$$
\frac{\partial \mathbf{K}_{u}^{(j)}}{\partial \rho_{e}}=\mathbf{k}_{e, \text { inc }}^{(j)}-\mathbf{k}_{e, \text { mat }}^{(j)}, \quad j=1,2, \ldots, n_{\text {load }}
$$

according to the defined multiple material interpolation model in (33), where $\mathbf{k}_{e, \text { inc }}^{(j)}$ and $\mathbf{k}_{e, \text { mat }}^{(j)}$ are the element stiffness matrices at the $j$-th load time calculated from using Young's moduli $E_{\mathrm{inc}}$ and $E_{\text {mat }}$, respectively.

\subsection{Extended BESO method}

The extended BESO method recently developed in [33] augments the original proposition in [56] through an additional damping treatment of sensitivity numbers, so as to improve the robustness and the effectiveness of the method, particularly in dealing with nonlinear designs with the presence of dissipative effects.

By the extended BESO method, the target volume of material usage $V^{(k)}$ at the current design iteration $(k$-th) is determined by

$$
V^{(k)}=\max \left\{V_{\text {req }},\left(1-c_{\mathrm{er}}\right) V^{(k-1)}\right\},
$$

in which the evolutionary ratio $c_{\mathrm{er}}$ determines the percentage of material to be removed from the design of the previous iteration. Once the final required material volume usage $V_{\text {req }}$ is reached, the optimization algorithm alters only the topology but keeps the volume fraction constant.

At each design iteration, the sensitivity numbers which denote the relative ranking of the element sensitivities are used to determine material phase exchange. When uniform meshes are used, the sensitivity number for the considered objective is defined as following using the element sensitivity computed from (53)

$$
\alpha_{e}=\left\{\begin{array}{cl}
\left(\frac{\partial J^{*}}{\partial \rho_{e}}\right)^{\eta}, & \text { for } \rho_{e}=1 \\
0, & \text { for } \rho_{e}=0 .
\end{array}\right.
$$

in which $\eta$ is a numerical damping coefficient (the same as the one applied in the Optimality Criteria method for density-based methods [61]). When $\eta=1$, we recover the conventional sensitivity numbers for linear elastic designs $[36,62]$. In the presence of dissipative effects, the sensitivity numbers vary by several orders of magnitude resulting in instabilities of the topology evolution process, especially when removing certain structural branches (see, e.g., [32, 33]). For this reason, the sensitivity numbers are damped in this work with " $\eta=0.5$ " as suggested in [33].

In order to avoid mesh-dependency and checkerboard patterns, sensitivity numbers are firstly smoothed by means of a filtering scheme [61]

$$
\alpha_{e}=\frac{\sum_{j=1}^{N_{\mathrm{e}}} w_{e j} \alpha_{j}}{\sum_{j=1}^{N_{\mathrm{e}}} w_{e j}}
$$


where $w_{e j}$ is a linear weight factor

$$
w_{e j}=\max \left(0, r_{\min }-\Delta(e, j)\right)
$$

determined according to the prescribed filter radius $r_{\min }$ and the element center-to-center distance $\Delta(e, j)$ between elements $\Omega_{e}$ and $\Omega_{j}$. Attention needs to be recalled that the filter (57) is also responsible for material exchange from the matrix phase $\left(\rho_{e}=0\right)$ to the inclusion phase $\left(\rho_{e}=1\right)$ by attributing filtered sensitivity number values to design variables that are associated to the matrix phase.

Due to the discrete nature of the BESO material model, the current sensitivity numbers need to be averaged with their historical information to improve the design convergence [35]

$$
\alpha_{e}^{(k)} \leftarrow \frac{\left(\alpha_{e}^{(k)}+\alpha_{e}^{(k-1)}\right)}{2} .
$$

The update of the topology design variables is realized by means of two threshold parameters $\alpha_{\text {del }}^{\text {th }}$ and $\alpha_{\text {add }}^{\text {th }}$ for material removal and addition, respectively $[32,56]$

$$
\rho_{e}^{(k+1)}= \begin{cases}0 & \text { if } \alpha_{e} \leq \alpha_{\mathrm{del}}^{\mathrm{th}} \text { and } \rho_{e}^{(k)}=1, \\ 1 & \text { if } \alpha_{\mathrm{add}}^{\mathrm{th}}<\alpha_{e} \text { and } \rho_{e}^{(k)}=0 \\ \rho_{e}^{(k)} & \text { otherwise. }\end{cases}
$$

The present scheme indicates that inclusion elements are exchanged to matrix elements when their sensitivity numbers are less than $\alpha_{\text {del }}^{\text {th }}$ and matrix elements are reversed to inclusion elements when their sensitivity numbers are greater than $\alpha_{\text {add }}^{\text {th }}$. The parameters $\alpha_{\text {del }}^{\text {th }}$ and $\alpha_{\text {add }}^{\text {th }}$ are obtained from the following iterative algorithm which was first proposed in [56] and recently adopted by the first author in $[32,33]$ :

1. Let $\alpha_{\text {add }}^{\text {th }}=\alpha_{\text {del }}^{\text {th }}=\alpha_{\text {th }}$, where the value $\alpha_{\text {th }}$ is determined iteratively such that the required material volume usage is met at the current iteration.

2. Compute the admission ratio $c_{\mathrm{ar}}$, which is defined as the volume of the recovered elements $\left(\rho_{e}=0 \rightarrow 1\right)$ divided by the total volume of the current design iteration. If $c_{\mathrm{ar}} \leq c_{\mathrm{ar}}^{\max }$, the maximum admission ratio, then skip the next steps; otherwise, $\alpha_{\text {del }}^{\text {th }}$ and $\alpha_{\text {add }}^{\text {th }}$ are redetermined in the next steps.

3. Determine $\alpha_{\text {add }}^{\text {th }}$ iteratively using only the sensitivity numbers of the matrix elements $\left(\rho_{e}=0\right)$ until the maximum admission ratio is met, i.e., $c_{\mathrm{ar}} \approx c_{\mathrm{ar}}^{\max }$.

4. Determine $\alpha_{\text {del }}^{\text {th }}$ iteratively using only the sensitivity numbers of the inclusion elements $\left(\rho_{e}=\right.$ 1) until the required material volume usage is met at the current iteration.

The introduction of $c_{\mathrm{ar}}^{\max }$ stabilizes the topology optimization process by controlling the number of elements reversed from matrix to inclusion. In the present work, $c_{\mathrm{ar}}^{\max }$ is set to a value greater than $1 \%$ so that it does not suppress the merit of the reverse procedure. 


\section{Numerical examples}

In this section, we show the performance of the proposed design framework through a series of $2 \mathrm{D}$ and $3 \mathrm{D}$ benchmark tests. In all 2D examples, uniform meshes of quadrilateral bilinear elements with the plane strain assumption are employed. Similarly, uniform mesh of eight-node cubic elements are adopted for the 3D design case. The same finite element discretization is adopted for both displacement and crack phase fields. The characteristic length scale parameter for the phase field regularization appearing in (1) is set to be twice that of the typical finite element size $\ell=2 h^{e}$. For the sake of clear visualization, only the crack phase field with the values over 0.4 is plotted. The material properties of the inclusion and the matrix phases are given in Table 1 according to [63].

With regard to topology optimization, all parameters involved in the extended BESO method as presented in Section 3.3 are held constant in all following examples. The evolutionary rate $c_{\mathrm{er}}$, which determines the relative percentage of material to be removed at each design iteration, is set to $c_{\mathrm{er}}=4 \%$ (exception for the third example $c_{\mathrm{er}}=6 \%$ ). The maximum admission ratio corresponding to the maximum percentage of recovered material that is allowed per iteration is set to $c_{\mathrm{ar}}^{\max }=2 \%$. The filter radius is set to be twice that of the typical finite element size $r_{\min }=2 h^{e}$.

Table 1: Material properties of the inclusion and the matrix [63].

\begin{tabular}{cccc}
\hline Name & Symbol & Value & Unit \\
\hline Young's modulus of inclusion & $E_{\text {inc }}$ & 52 & $\mathrm{GPa}$ \\
Young's modulus of matrix & $E_{\text {mat }}$ & 10.4 & $\mathrm{GPa}$ \\
Poisson's ratio of both phases & $v$ & 0.3 & {$[-]$} \\
Critical fracture stress of inclusion & $\sigma_{c, \text { inc }}$ & 30 & $\mathrm{MPa}$ \\
Critical fracture stress of matrix & $\sigma_{c, \text { mat }}$ & 10 & $\mathrm{MPa}$ \\
\hline
\end{tabular}

\subsection{Design of a $2 D$ reinforced plate with one pre-existing crack notch}

The problem setting of the 2D plate with one pre-existing crack notch is illustrated in Fig. 3(a). The dimensions of the plate is $50 \times 100 \mathrm{~mm}^{2}$. The whole plate is uniformly discretized into $60 \times 120$ square shaped bilinear elements. Plane strain assumption is assumed. The lower end of the plate is fixed vertically while free horizontally. The left bottom corner node is fixed in both directions to avoid rigid body motions. The upper end of the plate is prescribed with incremental displacement loads with $\Delta \overline{\mathbf{u}}=0.01 \mathrm{~mm}$ for the first five load increments and $\Delta \overline{\mathbf{u}}=0.002 \mathrm{~mm}$ for the following load increments. The incremental loading process continues until the reaction forces is below a prescribed criterion value indicating that the structure is completely broken.

Fig. 3(b) is the initial guess design with the inclusion phase occupying $10 \%$ of the domain area. By the extended BESO method, the inclusion phase area is gradually reduced to a target area fraction, $5 \%$ of the domain area. The pre-existing crack notch is simulated by prescribing Dirichlet conditions on the crack phase field with $d=1$ along the crack. The surrounding area of the initial crack notch (up to 2 times of the length scale parameter $\ell$ ) is treated as a non-designable region to avoid nonphysical designs with the inclusion material added within the already existing crack. The evolution of inclusion typologies together with their final crack patterns and the design 


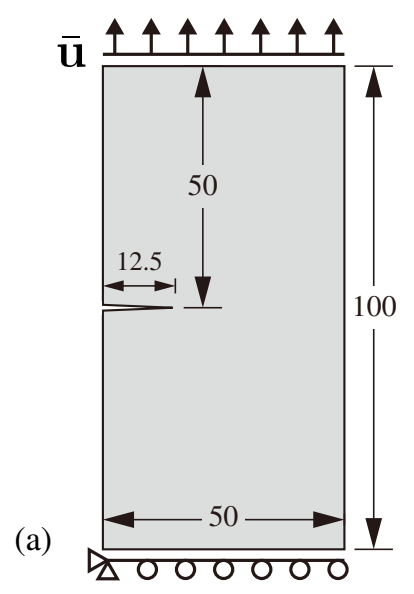

(b)

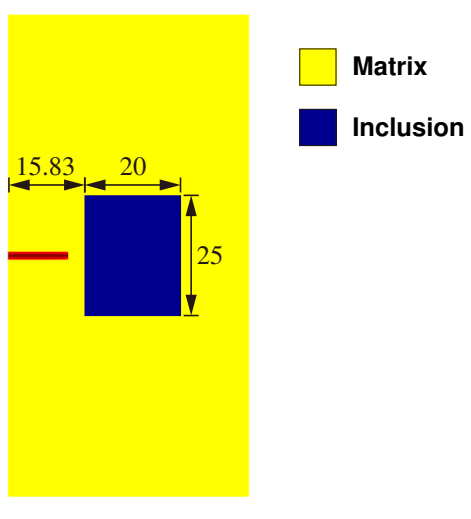

Figure 3: A 2D plate with one pre-existing crack notch subject to incremental traction loads: (a) problem depiction, (b) initial guess design.

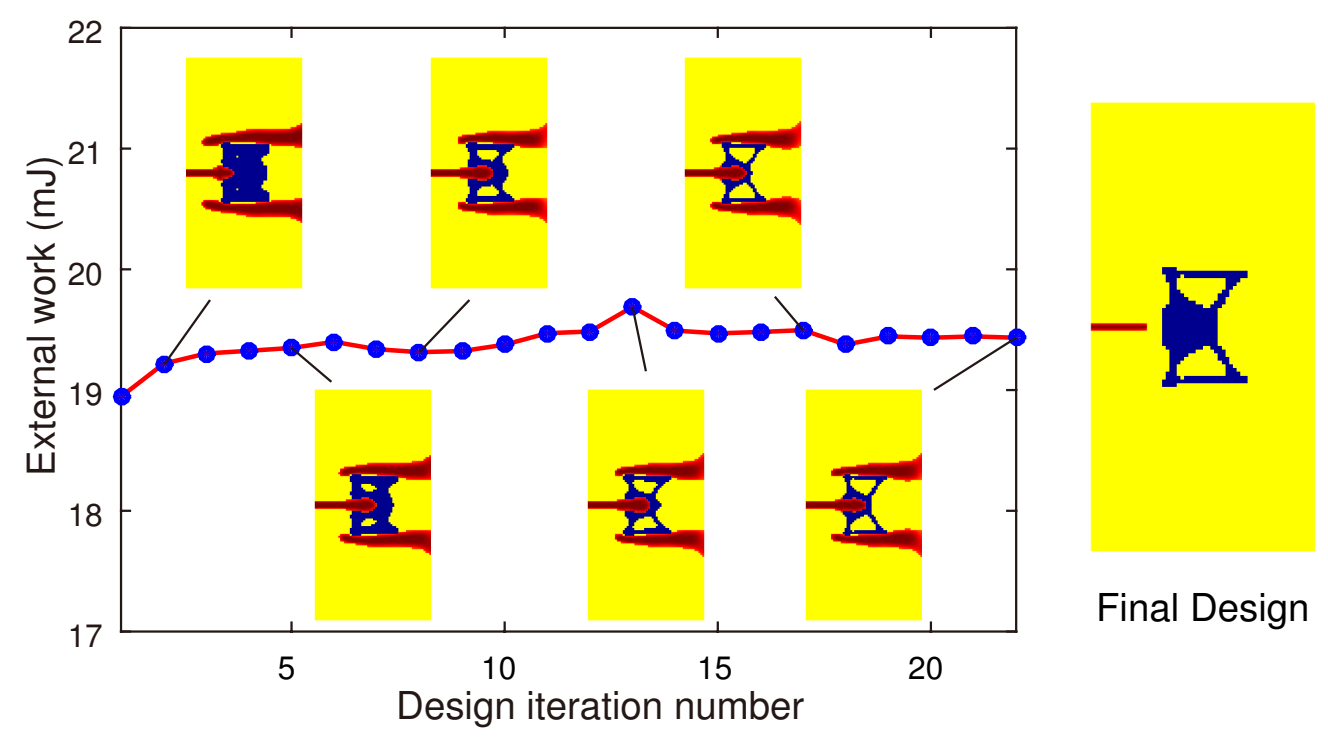

Figure 4: History of the evolution of inclusion typologies and their final crack patterns.

objective history are shown in Fig. 4. It can be observed from Fig. 4 that the fracture resistance of the composite structure gently improves whilst the area fraction of the inclusion phase gradually decreases from initial $10 \%$ to $5 \%$. It means that for the same fracture resistance performance, the required usage of inclusion phase can be largely saved via an optimal spatial distribution design.

Detailed propagation of the phase field crack of the optimally designed composite structure with one pre-existing crack notch subject to incremental traction loads is given in Fig. 5. The crack propagates into the inner supporting structure made of the inclusion phase during the initial incremental loads. Two other cracks initiate around the upper and lower left corners of the inner supporting structure and continue to propagate horizontally until the structure is fully broken.

The fracture resistance of the optimally designed composite structure is validated through a comparison study. Starting from the same initial guess design (Fig. 3(b)), topology optimization 


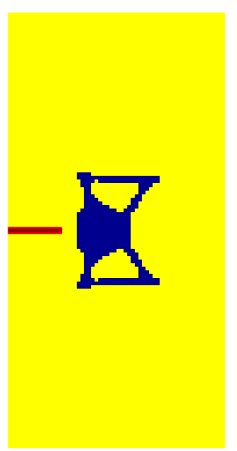

(a)

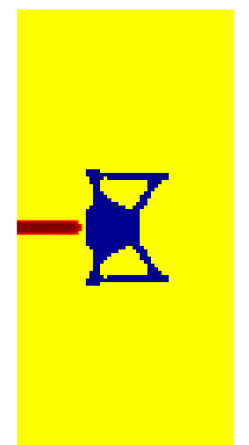

(b)

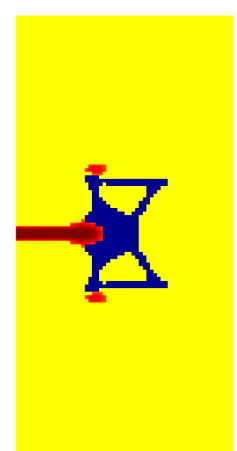

(c)

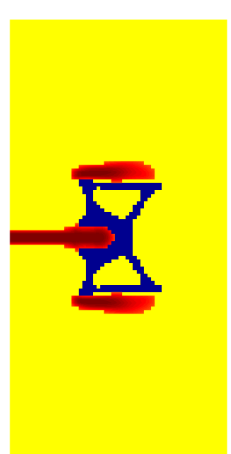

(d)

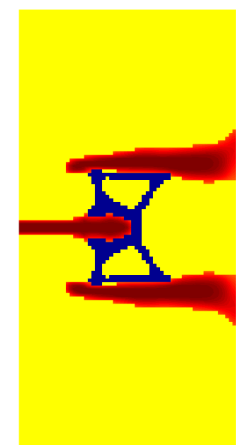

(e)

Figure 5: Crack propagation of the optimally designed composite structure with one pre-existing crack notch subject to incremental traction loads: (a) $\overline{\mathbf{u}}=0 \mathrm{~mm}$, (b) $\overline{\mathbf{u}}=0.060 \mathrm{~mm}$, (c) $\overline{\mathbf{u}}=0.076 \mathrm{~mm}$, (d)

$$
\overline{\mathbf{u}}=0.082 \mathrm{~mm} \text {, (e) } \overline{\mathbf{u}}=0.092 \mathrm{~mm} \text {. }
$$
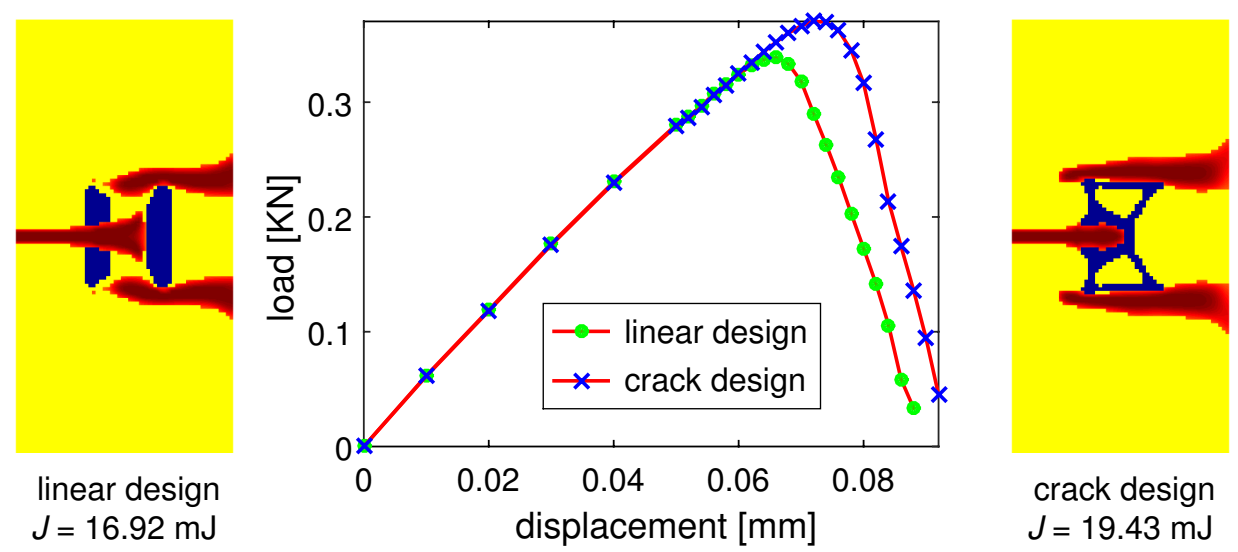

Figure 6: Fracture resistance comparison of two composite structures with one pre-existing crack notch subject to incremental traction loads.

using the same parameter setting has been carried out considering only linear elastic behavior without accounting for crack propagation, i.e. linear design, yielding two parallel bars along the vertical loading direction from the left design in Fig. 6. A complete fracturing simulation is carried out then on the linearly designed composite structure accounting for crack propagation. From both load-displacement curves and design objective values, the crack design is $15 \%$ more resistant to fracture than the linear design.

We would like to recall that the adopted BESO method is a heuristic scheme which does not necessarily guarantee a global optimum design. In the case of linear elasticity, the method has been proved to be insensitive to the two chosen initial starting topologies for structural stiffness maximization design [35]. However, such independency is not guaranteed when it comes to severe nonlinear problems as it is in the current case, i.e., different starting topologies may lead to different local optimum designs. Meanwhile, though the BESO method allows for both material removal and addition, their effectiveness are different. As stated in Section 3.3, sensitivity numbers are only evaluated for the inclusion elements and are set to zero for the matrix elements. It is only 


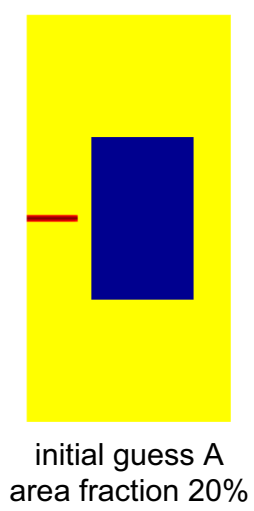

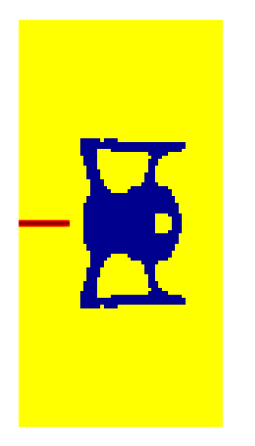

iteration 13

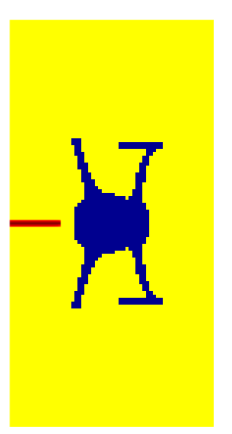

iteration 24

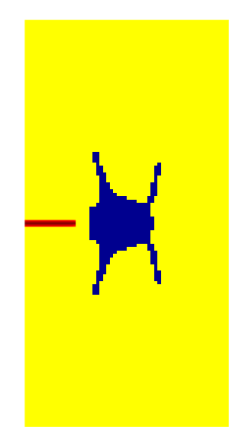

iteration 39

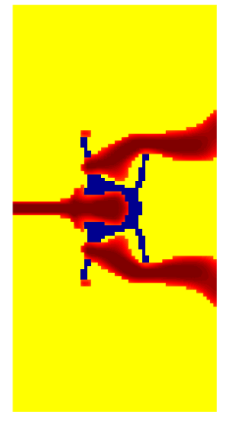

crack pattern $J=20.46 \mathrm{~mJ}$

Figure 7: Evolution of inclusion topologies from an inital guess design with the inclusion phase occupying $20 \%$ area fraction of the domain and the final crack pattern.
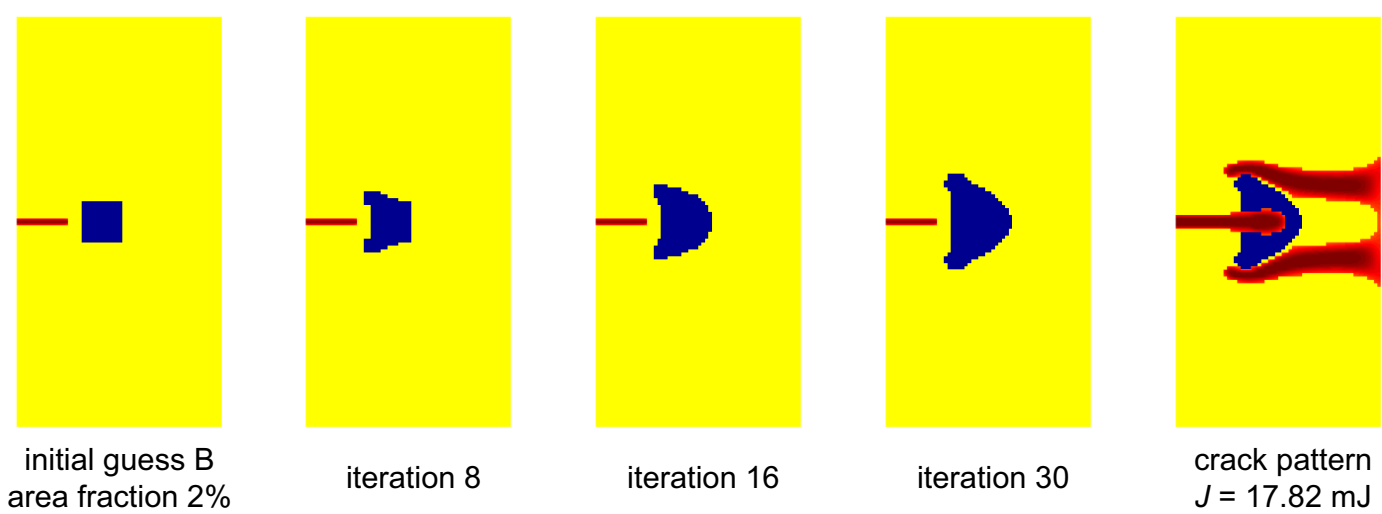

Figure 8: Evolution of inclusion topologies from an inital guess design with the inclusion phase occupying $2 \%$ area fraction of the domain and the final crack pattern.

due to the filtering scheme (57) that sensitivity numbers on the matrix elements neighboring to inclusion-matrix interface are evaluated. Therefore, it is in usual more effective to perform a gradual material reduction starting from a larger initial domain, meanwhile the recovery/addition serves as a complementary mechanism for minor adjustment [36].

For the purpose of comparison, we have redesigned the first example from two alternative initial topologies as shown in Figs. 7 and 8. For both cases, the resulted finial inclusion topologies are local optimum designs and are different from the previous design in Fig. 4. Note that in order to start with initial guess B with a lower inclusion area fraction, (57) needs to be modified for material addition and the maximum admission ration $\alpha_{\text {add }}^{\text {th }}$ should be exempted from the design. By comparing the values of the required work for complete fracture, a larger initial inclusion domain would result in a better design, however at the expense of more computing effort.

\subsection{Design of a $2 D$ reinforced plate with two pre-existing crack notches}

A 2D plate with two pre-existing crack notches in Fig. 9(a) is considered for design. Apart from the two pre-existing crack notches, the other problem settings are defined the same as the previous example. An initial guess design with the inclusion phase occupying $15 \%$ of the domain 


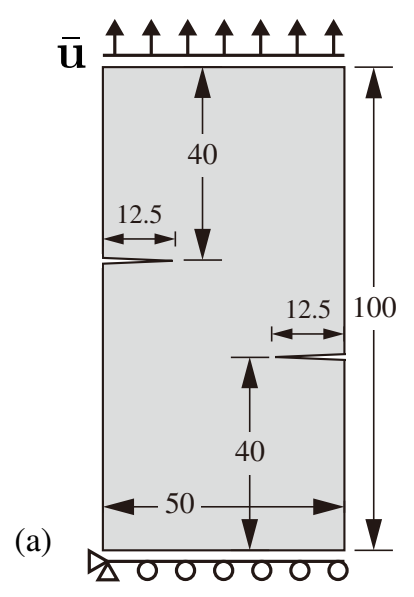

(b)

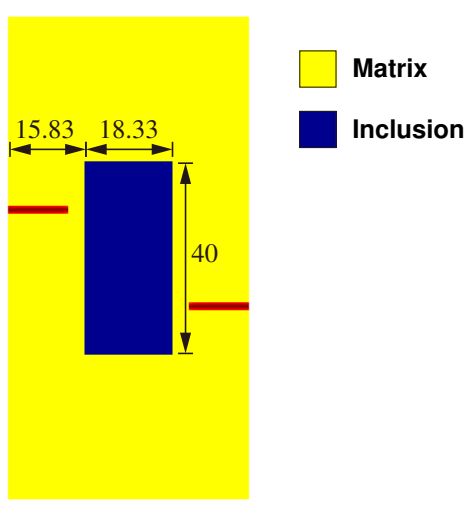

Figure 9: Illustration of a 2D plate with two pre-existing crack notches subject to incremental traction loads: (a) problem depiction, (b) initial guess design.

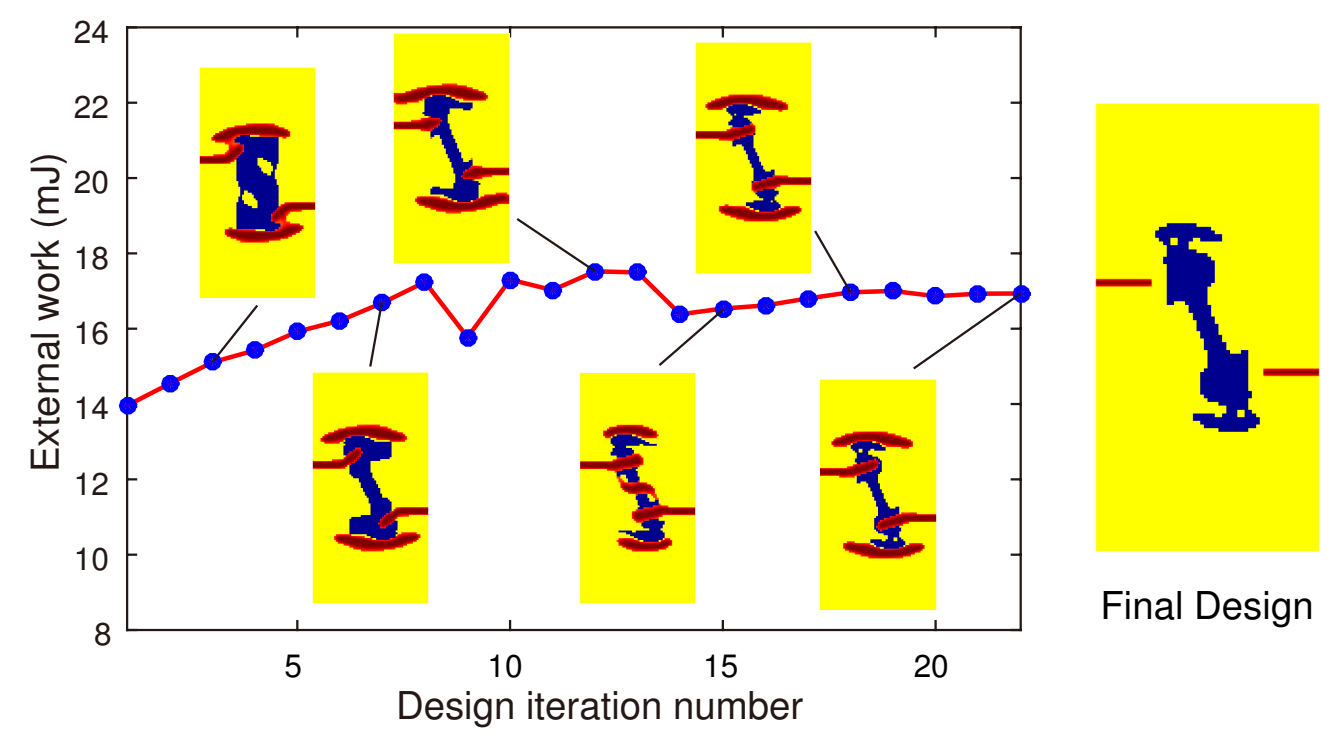

Figure 10: History of the evolution of inclusion typologies and their final crack patterns.

area is assumed as shown in Fig. 9(b). It is expected to optimally reduce the inclusion phase area fraction from $15 \%$ to $8 \%$ by using the developed method. The pre-existing two crack notches are simulated by prescribing Dirichlet conditions with $d=1$ along the crack. The surrounding area of the two initial crack notches is treated as a non-designable region to avoid nonphysical designs with the inclusion material added within the already existing crack. .

Fig. 10 shows the evolution of inclusion typologies together with their final crack patterns and the design objective history. Similar to the previous example, the fracture resistance of the composite structure gently improves whilst the area fraction of the inclusion gradually decreases from initial $14.67 \%$ to $8 \%$, indicating that for the same fracture resistance performance, the required usage of inclusion phase material can be largely saved via an optimal spatial distribution design. Due to the anti-symmetry of the problem setting, cracks appear anti-symmetrically in the upper 


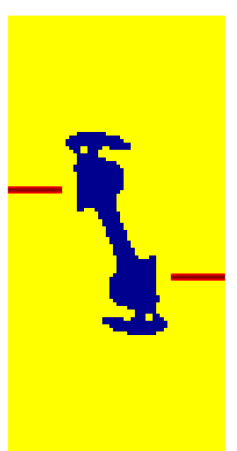

(a)

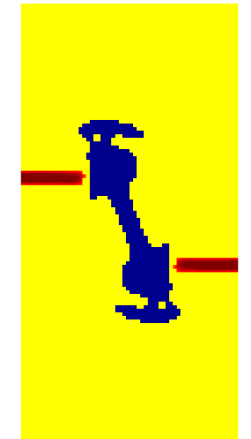

(b)

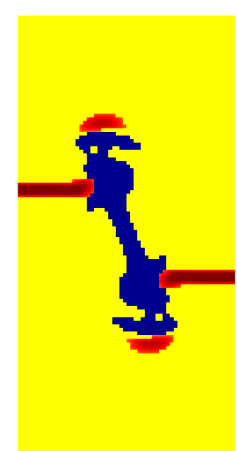

(c)

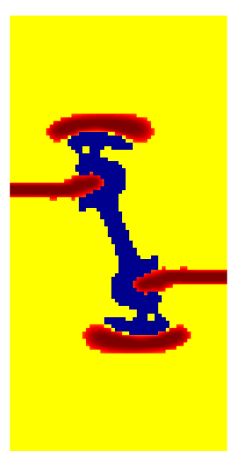

(d)

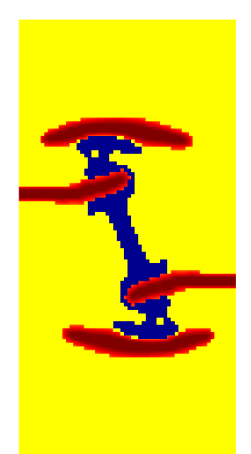

(e)

Figure 11: Crack propagation of the optimally designed composite structure with two pre-existing crack notches subject to incremental traction loads: (a) $\overline{\mathbf{u}}=0 \mathrm{~mm}$, (b) $\overline{\mathbf{u}}=0.060 \mathrm{~mm}$, (c) $\overline{\mathbf{u}}=0.072 \mathrm{~mm}$, (d)

$$
\overline{\mathbf{u}}=0.080 \mathrm{~mm},(\mathrm{e}) \overline{\mathbf{u}}=0.094 \mathrm{~mm} \text {. }
$$

and lower parts of the structure. Fig. 11 shows detailed propagation of the phase field crack of the optimally designed composite structure with two pre-existing crack notches subject to incremental traction loads. The two initial cracks propagate into the inner supporting structure made of the inclusion phase during the initial incremental loads. Then, two other cracks initiate at the upper and lower left surfaces of the inner supporting structure. All four cracks then continue to propagate until the structure is fully broken.

Similar to the previous example, a comparison study is also performed to validate the performance of the fracture resistance of the optimally designed composite structure. Topology optimization is carried out considering only linear elastic behavior without accounting for crack propagation starting from the same initial guess design (Fig. 9(b)) and using the same design parameters. Linear design without accounting for crack propagation results in two longer parallel bars compared to the linear design obtained in the previous example (due to increased inclusion usage) as shown in Fig. 12. The linearly designed composite structure is then subjected to a full fracturing simulation and its load-displacement curve is compared with the one of the crack design. From both load-displacement curves and design objective values, the fracture resistance of the crack design has been obviously increased for over $40 \%$ in comparison to the linear design.

\subsection{Design of a $2 D$ reinforced plate with multiple pre-existing cracks}

This example addresses a multi-objective design using the developed method to improve the fracture resistance of a $2 \mathrm{D}$ reinforced plate trying to accommodate the geometry of the inclusion to several different distributions of cracks at the same time to deal with possible random creation of cracks within the structures. For the sake of illustration, only 3 configurations are used here as shown in Fig. 13. All problem settings are defined the same as the previous two examples except for the use of a finer finite element discretization. Considering the simulation accuracy involving multiple cracks, a finer discretization with $100 \times 200$ square shaped bilinear elements is adopted in this design. Following the same design procedure as presented in the first two examples, the area fraction of inclusion is gradually reduced from initial $28 \%$ to $8 \%$. The initial distribution of inclusion phase is assumed to be a square shape enveloping all inner cracks of three cases as 

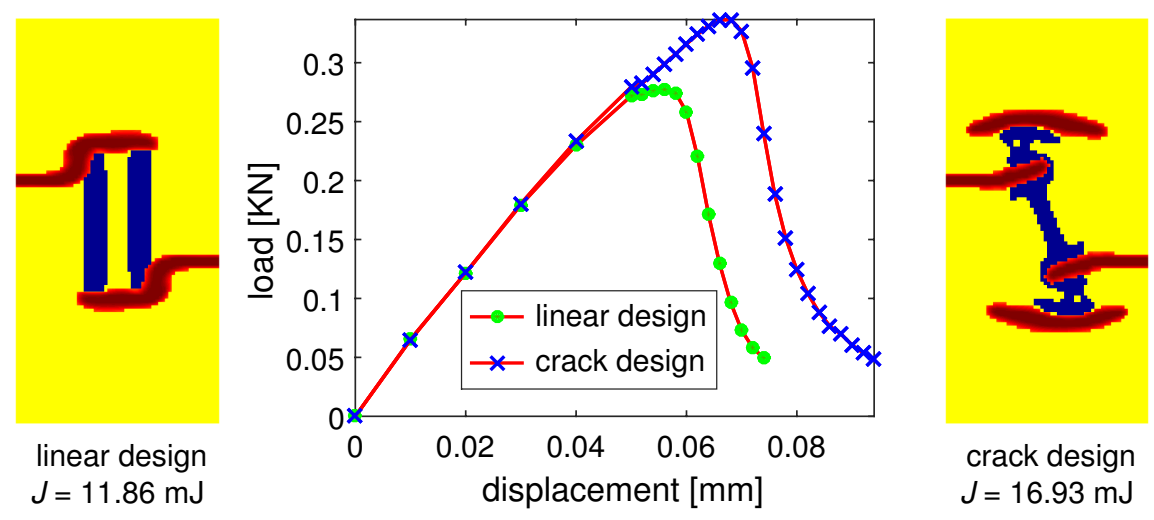

Figure 12: Fracture resistance comparison of two composite structures with two pre-existing crack notches subject to incremental traction loads.
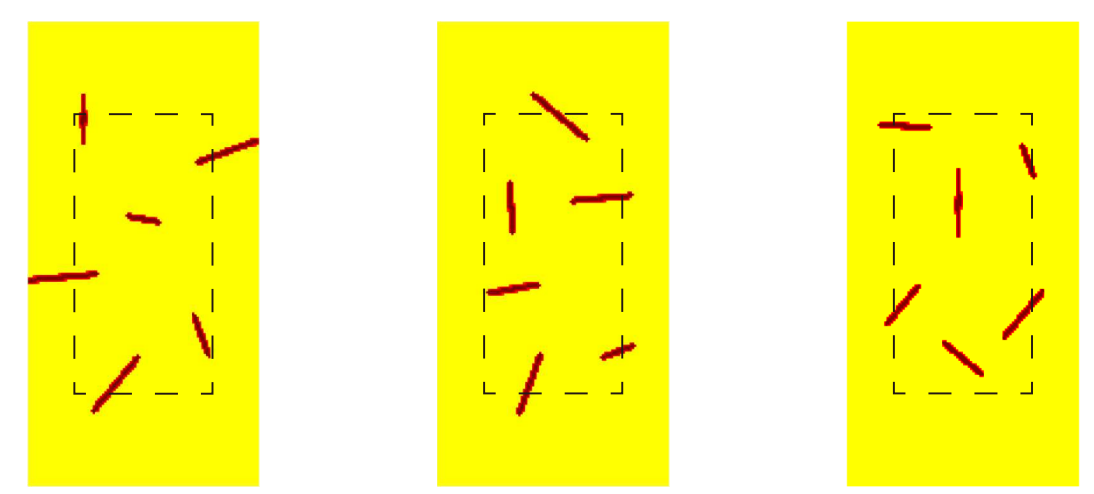

Figure 13: Three 2D plates with multiple pre-existing cracks (cases 1-3 from left to right).

shown by the dashed lines in Fig. 13.

The optimally designed distribution topology of inclusion phase is given in Fig. 14 and their final crack patterns are shown in Fig. 15. It can be observed that the inclusion phase is distributed preferably at places such that can prevent the further propagation of critical cracks. Attention needs to be recalled that due to the regularized description of cracks using the phase filed method, the surrounding region of all initial cracks is assumed to be non-designable. Otherwise the inclusion phase would fill all fictitious cracks by the design resulting in nonphysical designs.

\subsection{Design of a $3 D$ reinforced plate with one pre-existing crack notch surface}

A 3D plate with one pre-existing crack notch surface as shown in Fig. 16 is considered for design to further validate the developed method. The dimensions of the $3 \mathrm{D}$ plate is $50 \times 100 \times 6.67$ $\mathrm{mm}^{3}$. The whole volume domain is discretized into $60 \times 120 \times 8$ eight-node cubic elements. Similar to the $2 \mathrm{D}$ case in Section 4.1, the lower end of the plate is fixed vertically while free horizontally. The central node on the right end edge is fixed in all directions to avoid rigid body motions. The upper end of the plate is prescribed with incremental displacement loads with $\Delta \overline{\mathbf{u}}=0.01$ $\mathrm{mm}$ for first seven load increments and $\Delta \overline{\mathbf{u}}=0.002 \mathrm{~mm}$ for the following load increments. The incremental loading process continues until the reaction forces is below a prescribed criterion 

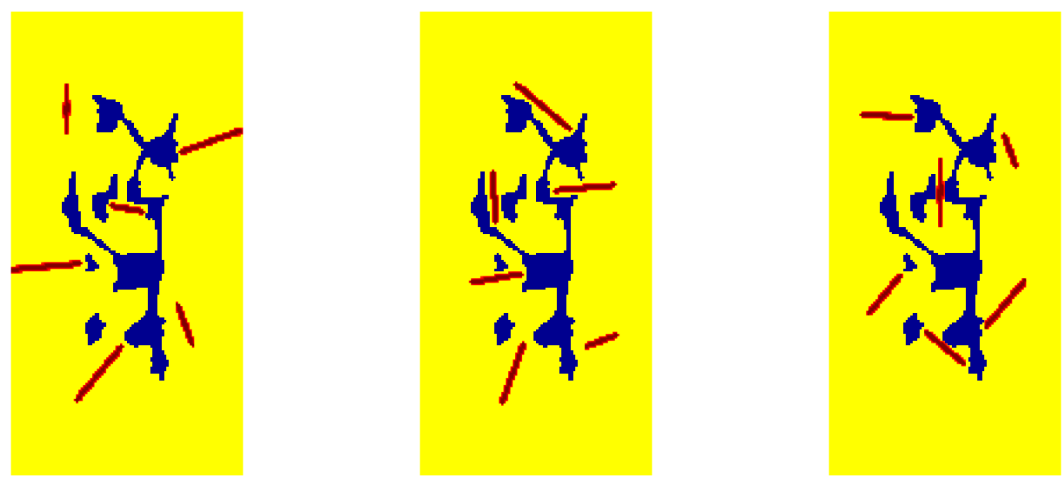

Figure 14: Optimally designed inclusion distribution topology for three 2D plates with multiple pre-existing cracks (cases 1-3 from left to right).

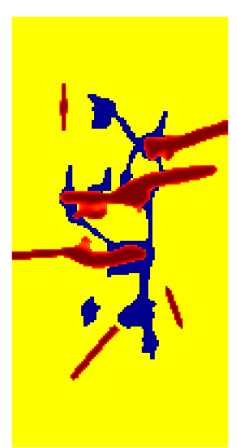

case 1

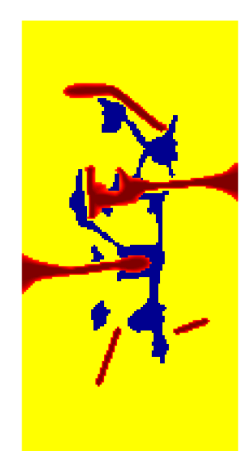

case 2

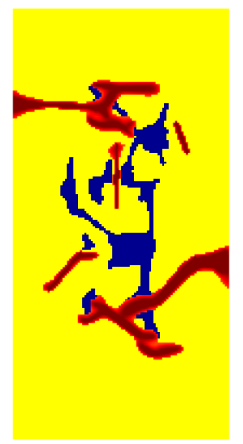

case 3

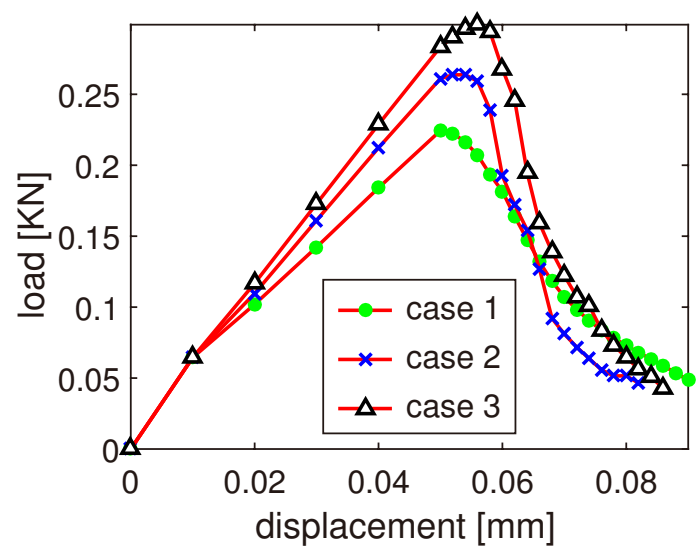

Figure 15: Final crack patterns of the three optimally designed 2D plates with multiple pre-existing cracks and their load-displacement curves.

value indicating that the structure is completely broken. The pre-existing crack notch surface is simulated by prescribing Dirichlet conditions $d=1$ along the crack surface. The surrounding volume of the initial crack surface (up to 2 times of the length scale parameter $\ell$ ) is treated as a non-designable region to avoid nonphysical designs.

Fig. 16(b) gives the initial guess design with the inclusion phase occupying $10 \%$ of the domain volume. By the developed method, the inclusion phase area is gradually reduced to the target volume fraction, $5 \%$ of the domain volume. The evolution of the spatial distribution topology of inclusion phase together with their final crack patterns and the design objective history are given in Fig. 17. The resultant hollow distribution topology design is similar to the one obtained in the 2D case in Section 4.1. It can be observed from Fig. 17 that unlike the 2D cases, the fracture resistance of the composite structure in the 3D case is obviously improved during the removal of material volume. From the $3 \mathrm{D}$ design, it is more obvious that for the same or even higher fracture resistance performance, the required usage of inclusion phase can be largely saved via an optimal spatial distribution design. This is because there exist much more inefficient material in the $3 \mathrm{D}$ case than the 2D case, such as those within the hollow, which could be clearly removed 


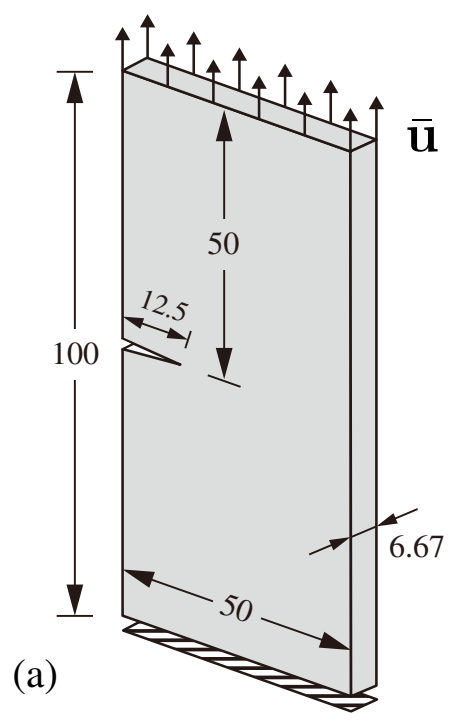

(b)

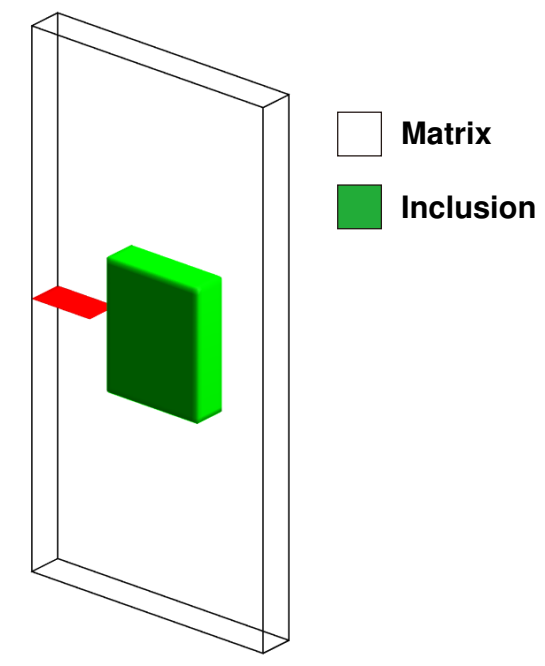

Figure 16: A 3D plate with one pre-existing crack notch subject to a traction load: (a) problem depiction, (b) initial guess design.

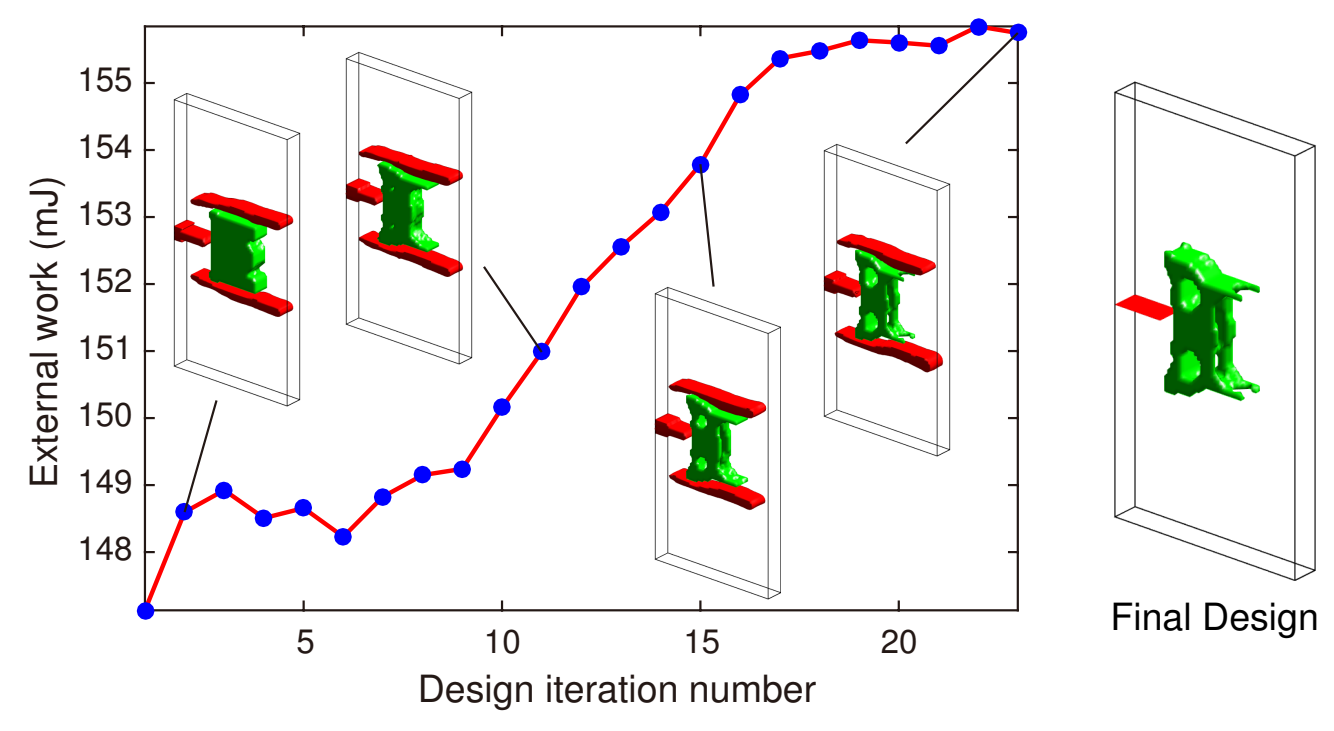

Figure 17: History of the evolution of inclusion typologies and their final crack patterns.

without weakening the fracture resistance of the composite structure. Detailed phase field crack propagation of the optimally designed 3D composite structure is given in Fig. 18, where the crack propagation trajectory is similar to the corresponding 2D case in Section 4.1.

\section{Conclusions}

In this paper, we have proposed a numerical design framework for fracture resistance of composites through an optimal design of the spatial distribution of inclusion phase. The phase field 


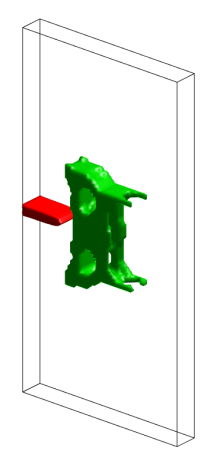

(a)

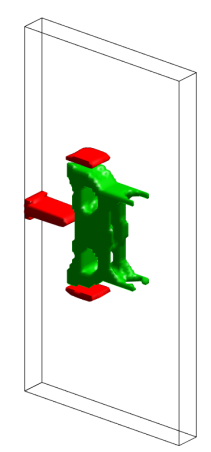

(b)

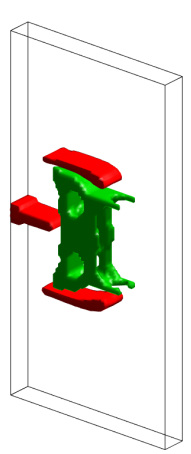

(c)

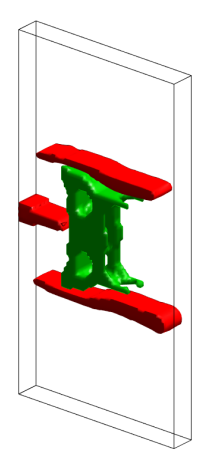

(d)

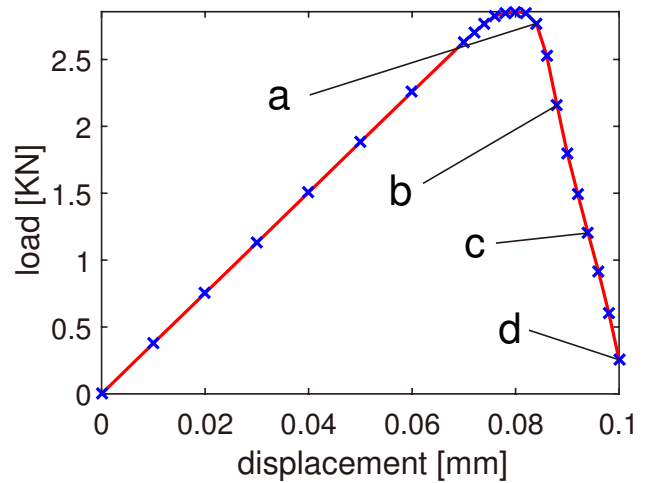

(e)

Figure 18: Crack propagation of the optimally designed 3D composite structure with one pre-existing crack notch subject to incremental traction loads: (a) $\overline{\mathbf{u}}=0.084 \mathrm{~mm}$, (b) $\overline{\mathbf{u}}=0.088 \mathrm{~mm}$, (c) $\overline{\mathbf{u}}=0.094 \mathrm{~mm}$, (d) $\overline{\mathbf{u}}=0.010 \mathrm{~mm}$, (e) load-displacement curve.

method to fracturing with a regularized description of discontinuity has been adopted for the modeling of complete fracturing process. The optimal design of the spatial distribution of inclusion phase is realized by means of topology optimization using an extend bi-directional evolutionary structural optimization method. Both 2D and 3D benchmark tests have been performed to validate the proposed design framework. It has been shown that significant improvement of the fracture resistance of composites has been achieved for designs accounting for full failure when compared to conventional linear designs.

Compared to previous studies in the literature on the subject, this work provides a much more efficient alternative for the design of high fracture resistant composites. There exist twofold merits of the developed design framework: on the one hand, the adoption of topology optimization provides uttermost design freedom, yielding higher fracture resistant designs; on the other hand, limited number of iterations is required for the design for the sake of using gradient information, which is of essential importance dealing with computationally demanding fracturing simulation.

\section{Acknowledgements}

This work is supported by the National Natural Science Foundation of China (51705165). This work has also benefited from a French government grant managed by ANR within the frame of the national program Investments for the Future ANR-11-LABX-022-01 (LABEX MMCD). JY thanks the financial support of Institut Universitaire de France (IUF).

\section{References}

[1] Y. Xu, T. You, Minimizing thermal residual stresses in ceramic matrix composites by using iterative mapreduce guided particle swarm optimization algorithm, Composite Structures 99 (2013) 388-396.

[2] Y. Xu, T. Gao, Optimizing thermal-elastic properties of C/CSiC composites using a hybrid approach and PSO algorithm, Materials 9 (4) (2016) 222.

[3] Z. Jing, X. Fan, Q. Sun, Stacking sequence optimization of composite laminates for maximum buckling load using permutation search algorithm, Composite Structures 121 (2015) 225-236. 
[4] Z. Jing, Q. Sun, V. V. Silberschmidt, A framework for design and optimization of tapered composite structures. Part I: From individual panel to global blending structure, Composite Structures 154 (2016) 106-128.

[5] M. Prechtel, G. Leugering, P. Steinmann, M. Stingl, Towards optimization of crack resistance of composite materials by adjustment of fiber shapes, Engineering Fracture Mechanics 78 (6) (2011) 944-960.

[6] G. X. Gu, L. Dimas, Z. Qin, M. J. Buehler, Optimization of composite fracture properties: Method, validation, and applications, Journal of Applied Mechanics 83 (7) (2016) 071006.

[7] B. San, H. Waisman, Optimization of carbon black polymer composite microstructure for rupture resistance, Journal of Applied Mechanics 84 (2) (2016) 021005.

[8] M. P. Bendsøe, N. Kikuchi, Generating optimal topologies in structural design using a homogenization method, Computer Methods in Applied Mechanics and Engineering 71 (2) (1988) 197-224.

[9] J. D. Deaton, R. V. Grandhi, A survey of structural and multidisciplinary continuum topology optimization: post 2000, Structural and Multidisciplinary Optimization 49 (1) (2014) 1-38.

[10] L. Xia, P. Breitkopf, Recent advances on topology optimization of multiscale nonlinear structures, Archives of Computational Methods in Engineering 24 (2) (2017) 227249.

[11] J. Zhu, W. Zhang, L. Xia, Topology optimization in aircraft and aerospace structures design, Archives of Computational Methods in Engineering 23 (4) (2015) 595-622.

[12] P. Duysinx, M. P. Bendsøe, Topology optimization of continuum structures with local stress constraints, International Journal for Numerical Methods in Engineering 43 (8) (1998) 1453-1478.

[13] C. Le, J. Norato, T. Bruns, C. Ha, D. Tortorelli, Stress-based topology optimization for continua, Structural and Multidisciplinary Optimization 41 (4) (2010) 605-620.

[14] X. Guo, W. Zhang, M. Wang, P. Wei, Stress-related topology optimization via level set approach, Computer Methods in Applied Mechanics and Engineering 200 (47-48) (2011) 3439-3452.

[15] Q. Xia, T. Shi, S. Liu, M. Wang, A level set solution to the stress-based structural shape and topology optimization, Computers and Structures 90-91 (2012) 55-64.

[16] M. Bruggi, P. Duysinx, Topology optimization for minimum weight with compliance and stress constraints, Structural and Multidisciplinary Optimization 46 (3) (2012) 369-384.

[17] Y. Luo, Z. Kang, Topology optimization of continuum structures with drucker-prager yield stress constraints, Computers and Structures 90 (2012) 65-75.

[18] Y. Luo, M. Y. Wang, Z. Kang, An enhanced aggregation method for topology optimization with local stress constraints, Computer Methods in Applied Mechanics and Engineering 254 (2013) 31-41.

[19] S. Cai, W. Zhang, J. Zhu, T. Gao, Stress constrained shape and topology optimization with fixed mesh: A b-spline finite cell method combined with level set function, Computer Methods in Applied Mechanics and Engineering 278 (2014) 361-387.

[20] S. Cai, W. Zhang, Stress constrained topology optimization with free-form design domains, Computer Methods in Applied Mechanics and Engineering 289 (2015) 267-290.

[21] V. J. Challis, A. P. Roberts, A. Wilkins, Fracture resistance via topology optimisation, Structural and Multidisciplinary Optimization 36 (3) (2008) 263-271.

[22] O. Amir, O. Sigmund, Reinforcement layout design for concrete structures based on continuum damage and truss topology optimization, Structural and Multidisciplinary Optimization 47 (2) (2012) 157-174.

[23] O. Amir, A topology optimization procedure for reinforced concrete structures, Computers and Structures 114 (2013) 46-58.

[24] M. Jansen, G. Lombaert, M. Schevenels, O. Sigmund, Topology optimization of fail-safe structures using a simplified local damage model, Structural and Multidisciplinary Optimization 49 (4) (2013) 657-666.

[25] K. A. James, H. Waisman, Failure mitigation in optimal topology design using a coupled nonlinear continuum damage model, Computer Methods in Applied Mechanics and Engineering 268 (2014) 614-631.

[26] Z. Kang, P. Liu, M. Li, Topology optimization considering fracture mechanics behaviors at specified locations, Structural and Multidisciplinary Optimization (2016) 1-18.

[27] P. Liu, Y. Luo, Z. Kang, Multi-material topology optimization considering interface behavior via xfem and level set method, Computer Methods in Applied Mechanics and Engineering 308 (2016) 113-133.

[28] C. F. Hilchenbach, E. Ramm, Optimization of multiphase structures considering damage, Structural and Multidisciplinary Optimization 51 (5) (2015) 1083-1096. 
[29] J. Kato, A. Lipka, E. Ramm, Multiphase material optimization for fiber reinforced composites with strain softening, Structural and Multidisciplinary Optimization 39 (1) (2009) 63-81.

[30] J. Kato, E. Ramm, Multiphase layout optimization for fiber reinforced composites considering a damage model, Engineering Structures 49 (2013) 202-220.

[31] L. Li, K. Khandelwal, Design of fracture resistant energy absorbing structures using elastoplastic topology optimization, Structural and Multidisciplinary Optimization (2017) 1-29.

[32] F. Fritzen, L. Xia, M. Leuschner, P. Breitkopf, Topology optimization of multiscale elastoviscoplastic structures, International Journal for Numerical Methods in Engineering 106 (6) (2016) 430-453.

[33] L. Xia, F. Fritzen, P. Breitkopf, Evolutionary topology optimization of elastoplastic structures, Structural and Multidisciplinary Optimization 55 (2) (2017) 569-581.

[34] Y. M. Xie, G. P. Steven, A simple evolutionary procedure for structural optimization, Computers and Structures 49 (5) (1993) 885-896.

[35] X. Huang, Y. M. Xie, Convergent and mesh-independent solutions for the bi-directional evolutionary structural optimization method, Finite Elements in Analysis and Design 43 (14) (2007) 1039-1049.

[36] L. Xia, Q. Xia, X. Huang, Y. Xie, Bi-directional evolutionary structural optimization on advanced structures and materials: A comprehensive review, Archives of Computational Methods in Engineeringdoi:10.1007/s11831016-9203-2.

[37] L. Xia, P. Breitkopf, Concurrent topology optimization design of material and structure within $\mathrm{FE}^{2}$ nonlinear multiscale analysis framework, Computer Methods in Applied Mechanics and Engineering, 278 (2014) 524542.

[38] L. Xia, P. Breitkopf, Multiscale structural topology optimization with an approximate constitutive model for local material microstructure, Computer Methods in Applied Mechanics and Engineering, 286 (2015) 147-167.

[39] G. Francfort, J. Marigo, Revisiting brittle fracture as an energy minimization problem, Journal of the Mechanics and Physics of Solids 46 (8) (1998) 1319-1342.

[40] B. Bourdin, G. Francfort, J. Marigo, The variational approach to fracture, Journal of elasticity 91 (1-3) (2008) 5-148.

[41] V. Hakim, A. Karma, Laws of crack motion and phase-field models of fracture, Journal of the Mechanics and Physics of Solids 57 (2) (2009) 342-368.

[42] C. Miehe, F. Welschinger, M. Hofacker, Thermodynamically consistent phase-field models of fracture: Variational principles and multi-field fe implementations, International Journal for Numerical Methods in Engineering 83 (2010) 1273-1311.

[43] C. Miehe, M. Hofacker, F. Welschinger, A phase field model for rate-independent crack propagation: Robust algorithmic implementation based on operator splits, Computer Methods in Applied Mechanics and Engineering 199 (45-48) (2010) 2765-2778.

[44] M. Borden, C. Verhoosel, M. Scott, T. Hughes, C. Landis, A phase-field description of dynamic brittle fracture, Computer Methods in Applied Mechanics and Engineering 217-220 (2012) 77-95.

[45] C. Miehe, L.-M. Schanzel, H. Ulmer, Phase field modeling of fracture in multi-physics problems. Part I. Balance of crack surface and failure criteria for brittle crack propagation in thermo-elastic solids, Computer Methods in Applied Mechanics and Engineering 294 (2015) 449-485.

[46] T. Nguyen, J. Yvonnet, Q.-Z. Zhu, M. Bornert, C. Chateau, A phase field method to simulate crack nucleation and propagation in strongly heterogeneous materials from direct imaging of their microstructure, Engineering Fracture Mechanics 139 (2015) 18-39.

[47] T. Nguyen, J. Yvonnet, M. Bornert, C. Chateau, K. Sab, R. Romani, R. Le Roy, On the choice of parameters in the phase field method for simulating crack initiation with experimental validation, International Journal of Fracture 197 (2016) 213-226.

[48] T. Nguyen, J. Yvonnet, M. Bornert, C. Chateau, Initiation and propagation of complex 3D networks of cracks in heterogeneous quasi-brittle materials: Direct comparison between in situ testing-microCT experiments and phase field simulations, Journal of the Mechanics and Physics of Solids 99 (2016) 320-350.

[49] D. Mumford, J. Shah, Optimal approximations by piecewise smooth functions and associated variational problems, Communications on Pure and Applied Mathematics 42 (5) (1989) 577-685.

[50] L. Ambrosio, V. Tortorelli, Approximation of functional depending on jumps by elliptic functional via $\Gamma$ - 
convergence, Communications on Pure and Applied Mathematics 43 (8) (1990) 999-1036.

[51] C. Miehe, M. Hofacker, L.-M. Schanzel, F. Aldakheel, Phase field modeling of fracture in multi-physics problems. Part II. Coupled brittle-to-ductile failure criteria and crack propagation in thermo-elastic-plastic solids, Computer Methods in Applied Mechanics and Engineering 294 (2015) 486-522.

[52] C. Miehe, S. Mauthe, Phase field modeling of fracture in multi-physics problems. Part III. Crack driving forces in hydro-poro-elasticity and hydraulic fracturing of fluid-saturated porous media, Computer Methods in Applied Mechanics and Engineering 304 (2016) 619-655.

[53] X. Huang, Y. M. Xie, Bi-directional evolutionary topology optimization of continuum structures with one or multiple materials, Computational Mechanics 43 (3) (2009) 393-401.

[54] K. Maute, S. Schwarz, E. Ramm, Adaptive topology optimization of elastoplastic structures, Structural Optimization 15 (2) (1998) 81-91.

[55] S. Schwarz, K. Maute, E. Ramm, Topology and shape optimization for elastoplastic structural response, Computer Methods in Applied Mechanics and Engineering 190 (15-17) (2001) 2135-2155.

[56] X. Huang, Y. M. Xie, Topology optimization of nonlinear structures under displacement loading, Engineering Structures 30 (7) (2008) 2057-2068.

[57] M. Bogomolny, O. Amir, Conceptual design of reinforced concrete structures using topology optimization with elastoplastic material modeling, International Journal for Numerical Methods in Engineering 90 (13) (2012) $1578-1597$.

[58] J. Kato, H. Hoshiba, S. Takase, K. Terada, T. Kyoya, Analytical sensitivity in topology optimization for elastoplastic composites, Structural and Multidisciplinary Optimization 52 (3) (2015) 507-526.

[59] T. Buhl, C. Pedersen, O. Sigmund, Stiffness design of geometrically nonlinear structures using topology optimization, Structural and Multidisciplinary Optimization 19 (2) (2000) 93-104.

[60] S. Cho, H.-S. Jung, Design sensitivity analysis and topology optimization of displacement-loaded non-linear structures, Computer Methods in Applied Mechanics and Engineering 192 (22-23) (2003) 2539-2553.

[61] O. Sigmund, A 99 line topology optimization code written in Matlab, Structural and Multidisciplinary Optimization 21 (2) (2001) 120-127.

[62] X. Huang, Y. M. Xie, Topology Optimization of Continuum Structures: Methods and Applications, John Wiley \& Sons, Chichester, 2010.

[63] T. Nguyen, J. Yvonnet, Q.-Z. Zhu, M. Bornert, C. Chateau, A phase-field method for computational modeling of interfacial damage interacting with crack propagation in realistic microstructures obtained by microtomography, Computer Methods in Applied Mechanics and Engineering 312 (2016) 567-595. 